\title{
Nonideal Sampling and Interpolation From Noisy Observations in Shift-Invariant Spaces
}

\author{
Yonina C. Eldar, Member, IEEE, and Michael Unser, Fellow, IEEE
}

\begin{abstract}
Digital analysis and processing of signals inherently relies on the existence of methods for reconstructing a continuoustime signal from a sequence of corrupted discrete-time samples. In this paper, a general formulation of this problem is developed that treats the interpolation problem from ideal, noisy samples, and the deconvolution problem in which the signal is filtered prior to sampling, in a unified way. The signal reconstruction is performed in a shift-invariant subspace spanned by the integer shifts of a generating function, where the expansion coefficients are obtained by processing the noisy samples with a digital correction filter. Several alternative approaches to designing the correction filter are suggested, which differ in their assumptions on the signal and noise. The classical deconvolution solutions (least-squares, Tikhonov, and Wiener) are adapted to our particular situation, and new methods that are optimal in a minimax sense are also proposed. The solutions often have a similar structure and can be computed simply and efficiently by digital filtering. Some concrete examples of reconstruction filters are presented, as well as simple guidelines for selecting the free parameters (e.g., regularization) of the various algorithms.
\end{abstract}

Index Terms-Deconvolution, interpolation, minimax reconstruction, sampling.

\section{INTRODUCTION}

$\mathbf{S}$ GNAL deconvolution is aimed at removing the system response effect on a signal, and is prevalent in a vast area of applications such as communications, imaging, and speech processing among others. Deconvolution can be cast in the framework of estimation in linear models, a topic that has been studied extensively in the past century following the classical works of Wiener [1] and Kolmogorov [2]. A fundamental problem they treated is that of estimating a stationary random signal in additive stationary noise, where the signal is convolved with a linear time-invariant (LTI) system. The reconstruction strategy is based on filtering the noisy signal with an LTI estimation filter with the goal of undoing the system influence and mitigating the noise.

In the context of sampling [3]-[5], a natural extension of the classical linear estimation problem is that of reconstructing a continuous-time function given a sequence of corrupted discrete measurements. When the measurements are samples of an

\footnotetext{
Manuscript received December 9, 2004; revised July 22, 2005. This work was supported in part by the European Union's Human Potential Programme, under the contract HPRN-CT-2003-00285 (HASSIP), and by the Israel Science Foundation under Grant no. 153104. The associate editor coordinating the review of this manuscript and approving it for publication was Prof. Karim Drouiche.

Y. Eldar is with the Technion-Israel Institute of Technology, Haifa 32000, Israel (e-mail: yonina@ee.technion.ac.il).

M. Unser is with the Biomedical Imaging Group, Ecole Polytechnique Fédérale de Lausanne (EPFL), CH-1015 Lausanne, Switzerland (e-mail: michael.unser@epfl.ch).

Digital Object Identifier 10.1109/TSP.2006.873365
}

underlying continuous-time signal, we obtain an interpolation problem from noisy data [6], [7]. A more general formulation of this problem allows for nonideal samples in which the continuous-time signal is filtered prior to ideal sampling, resulting in a deconvolution problem with the distinctive feature that we are seeking a continuous-time solution.

In this paper, we develop a unified approach to this type of reconstruction that extends some of the noise-free sampling and reconstruction techniques that have been investigated in the literature [8]-[12]. The specific problem we treat is that of reconstructing a continuous-time signal $x(t)$ from noisy, nonideal samples, that are modeled as the ideal uniform samples of the output of a sampling filter with impulse response $\tilde{\varphi}(-t)$, with $x(t)$ as its input. We seek a continuous-time reconstruction $\hat{x}(t)$ which is constrained to lie in a given shift-invariant space $\mathcal{V}=$ $\operatorname{span}\{\varphi(t-n)\}_{n \in \mathbb{Z}}$, spanned by integer shifts of a reconstruction filter $\varphi(t)$ [13], [14]. The reconstruction is obtained by filtering the noisy samples by a discrete-time filter $h[n]$ prior to reconstruction with the filter $\varphi(t)$. Our approach is general in that we allow for arbitrary sampling and reconstruction filters.

The two main reconstruction strategies in the noise-free case are consistent reconstruction [8]-[10] and minimax reconstruction [12]. The consistent approach is based on fitting the data exactly, so that $\hat{x}(t)$ yields the same samples as $x(t)$. Under an appropriate condition on the filters, the reconstruction is unique and is equal to the oblique projection $\hat{x}(t)=E_{\mathcal{V} \widetilde{\mathcal{V}} \perp} x(t)$ onto the reconstruction space $\mathcal{V}$ along the orthogonal complement of the sampling space $\widetilde{\mathcal{V}}=\operatorname{span}\{\tilde{\varphi}(t-n)\}_{n \in \mathbb{Z}}$. The minimax reconstruction is designed to minimize the worst-case squared-norm of the error between $\hat{x}(t)$ and $P_{\mathcal{V}} x(t)$ and can be written as the double projection $\hat{x}(t)=P_{\mathcal{V}} P_{\widetilde{\mathcal{V}}} x(t)$, where $P_{\mathcal{V}}$ and $P_{\widetilde{\mathcal{V}}}$ are the orthogonal projections onto $\mathcal{V}$ and $\widetilde{\mathcal{V}}$, respectively.

Sampling in the presence of noise has been investigated primarily in the context of bandlimited sampling. Iterative reconstruction algorithms in a general shift-invariant setting are considered in [14]. The work in this area includes analysis of the noise effects in existing reconstruction systems, e.g., [15]-[18] and references therein, and concrete reconstruction methods from noisy samples, such as [14], and [19]-[21]. However, the proposed algorithms tend to focus on the bandlimited setting and are typically not specified to be optimal from the point of view of statistical estimation theory.

Here, we suggest several noniterative reconstruction strategies that differ in their assumptions on the input signal and the noise. Three alternative formulations of the problem are developed: 1) deterministic (least-squares, Tikhonov), 2) stochastic (Wiener), and 3) mixed, where the signal is assumed to be deterministic and the noise random (minimax mean-squared error 
(MSE)). As we will see, the first setting leads to a generalization of the consistent strategy, while the second and third lead to extensions of the minimax solution. Except for the least-squares method, which is completely data driven, the common denominator between these various approaches is that they take advantage of some a priori knowledge on the class of underlying signals and that they constrain the solution accordingly. Depending on the type of formulation, these can be given in the form of a regularization constraint (Tikhonov), the inclusion into some particular smoothness class (minimax), or a precise specification of the power spectrum of the signal (Wiener). Interestingly, the solutions obtained are often similar and can be computed simply and efficiently by digital filtering.

Our development is pragmatic in the sense that we suggest a panorama of solutions, emphasizing commonalities and equivalence in order to help the practitioner design the most appropriate filter for his particular application. This approach provides a number of different routes (and formulations) that in many cases lead to the same computational solution, while providing several complementary insights into the problem as well as on the very notion of optimality.

The paper is organized as follows. In Section II, we describe our problem and introduce some mathematical preliminaries. In Section III, we formulate different design criteria and derive the corresponding solutions. This leads to the specification of four primary families of reconstruction filters, three of which (inverse filter, regularized least-squares or Tikhonov, and Wiener) are adaptations of classical deconvolution methods (cf. [22]-[24]) to our particular setting. Our fourth minimax solution is less conventional in the sense that it considers a mixed setting where the signal is deterministic and the measurement noise stochastic, and is based on our prior work on minimax estimation [25], [26]. A comparison of the various techniques is presented in Section IV, which is based on the analysis of some special cases, including the classical setting of a bandlimited reconstruction. Finally, to illustrate the generality of our formulation, we consider several concrete examples of nonbandlimited reconstructions in Section V. In the process, we provide a general computational method for determining the rational form of the transfer function ( $z$-transform) of the various filters for a reconstruction in a given spline space where the basis functions are compactly supported. The fact that we can display such rational solutions, which are generally not available in the bandlimited case nor in the traditional formulation of the Wiener filter, is interesting from a practical perspective because it naturally yields low complexity recursive algorithms (infinite-impulse-response (IIR) filters).

\section{PReliminaries AND Reconstruction Problem}

Throughout this paper, parentheses are used for continuous-time signals, e.g., $x(t)$, and brackets for discrete-time signals, e.g., $x[n]$. The continuous-time Fourier transform of a signal $x(t) \in L_{1}$ is denoted by $X(f)=\int_{-\infty}^{+\infty} x(t) e^{-j 2 \pi f t} d t$, and the discrete-time Fourier transform of a sequence $y[n] \in \ell_{1}$ is denoted by $Y\left(e^{j 2 \pi f}\right)$, where $Y(z)=\sum_{n \in \mathbb{Z}} y[n] z^{-n}$ is the $z$-transform of $y[n]$, and $\mathbb{Z}$ denotes the integers. These definitions can also be extended to finite energy signals

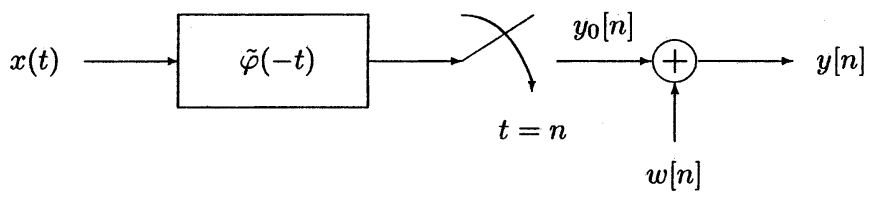

Fig. 1. Measurement model.

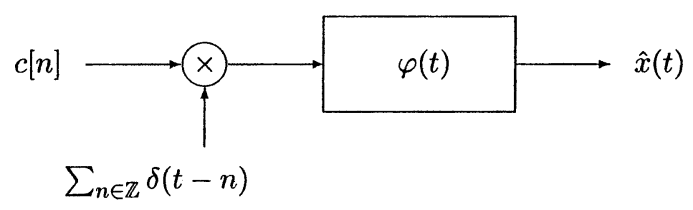

Fig. 2. Reconstruction model.

(i.e., $x(t) \in L_{2}$ and $y[n] \in \ell_{2}$ ). The standard $L_{2}$-inner product between two real signals $\tilde{\varphi}(t)$ and $x(t)$ is written as $\langle\tilde{\varphi}(t), x(t)\rangle=\int_{-\infty}^{+\infty} x(t) \tilde{\varphi}(t) d t$, and the discrete-time convolution between the sequences $h[n]$ and $x[n]$ is denoted by $(h * x)[n]=\sum_{m \in \mathbb{Z}} h[m] x[n-m]$. The shift-invariant spaces spanned by the functions $\varphi(t)$ and $\tilde{\varphi}(t)$ are denoted by $\mathcal{V}=\operatorname{span}\{\varphi(t-n)\}_{n \in \mathbb{Z}}$ and $\tilde{\mathcal{V}}=\operatorname{span}\{\tilde{\varphi}(t-n)\}_{n \in \mathbb{Z}}$, respectively, and $P_{\mathcal{V}}$ is the orthogonal projection onto the space $\mathcal{V}$

The basic problem we treat is the recovery of a continuoustime signal $x(t)$ given some equally spaced, noisy measurements $y[n]$. The signal $x(t)$ can either be deterministic, or a zero-mean stationary signal with known power spectrum. The sampling of the signal is nonideal in the sense that it is first convolved with $\tilde{h}(t)=\tilde{\varphi}(-t)$; typically, the impulse response of a measurement device. Moreover, it is corrupted by discrete stationary additive noise $w[n]$ with zero mean and known correlation function. Noting that the samples at times $t=n$ of the convolution $x(t) * \tilde{\varphi}(-t)$ can be expressed as the inner product sequence $\langle\tilde{\varphi}(t-n), x(t)\rangle$, we can write the measurements $y[n]$ as

$$
y[n]=\langle\tilde{\varphi}(t-n), x(t)\rangle+w[n], \quad n \in \mathbb{Z} .
$$

The measurement model is depicted in Fig. 1, in which $y_{0}[n]=$ $\langle\tilde{\varphi}(t-n), x(t)\rangle$ denote the noise-free samples.

When the sampling is ideal, i.e., when $\tilde{\varphi}(t)=\delta(t)$, we have an interpolation problem with noisy data; otherwise, a deconvolution problem with the distinctive feature that we are seeking a continuous-time solution. Since we have only a countable set of measurements and the unknown signal $x(t)$ is defined over a continuum, the reconstruction problem is inherently ill-posed. To resolve this ambiguity, we seek a reconstruction $\hat{x}(t)$ that is included in some "shift-invariant" Hilbert space $\mathcal{V}$, spanned by the integer shifts of a generating function $\varphi(t)$ [13], [14]. The signal reconstruction (estimate) is therefore constrained to be of the form

$$
\hat{x}(t)=\sum_{n \in \mathbb{Z}} c[n] \varphi(t-n)
$$

and is specified in terms of the unknowns $c[n]$. The reconstruction $\hat{x}(t)$ can be obtained by modulating the sequence $c[n]$ by an impulse train $\sum_{n \in \mathbb{Z}} \delta(t-n)$, and then filtering the modulated impulse train by a filter with impulse response $\varphi(t)$, as depicted in Fig. 2. 


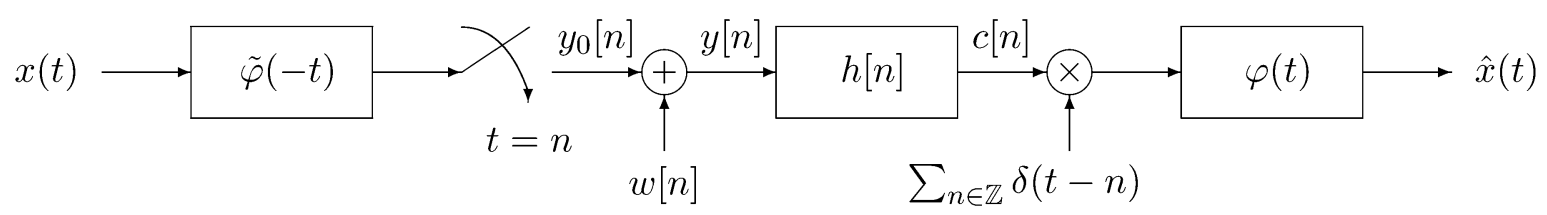

Fig. 3. Sampling and reconstruction scheme.

Given a sampling filter $\tilde{\varphi}(-t)$ and a reconstruction filter $\varphi(t)$, we reconstruct $x(t)$ from the samples $y[n]$ by first applying a digital correction filter, as illustrated in Fig. 3. The problem then is to design the filter $h[n]$ such that $\hat{x}(t)$ is close to $x(t)$ in some sense, where $\hat{x}(t)$ is specified by (2), with expansion coefficients $c[n]$ determined as

$$
c[n]=(h * y)[n]
$$

By appropriately choosing the frequency response $H\left(e^{j 2 \pi f}\right)$ of the filter $h[n]$ in Fig. 3, the two noise-free reconstruction strategies-i.e., consistent and minimax reconstruction—can be cast in this framework. Specifically, denoting by $\widetilde{\Phi}(f)$ and $\Phi(f)$ the Fourier transforms of $\tilde{\varphi}(t)$ and $\varphi(t)$, respectively, consistent reconstruction [8] is obtained with the filter

$$
H_{\mathrm{con}}\left(e^{j 2 \pi f}\right)=\frac{1}{\sum_{k \in \mathbb{Z}} \widetilde{\Phi}^{*}(f+k) \Phi(f+k)}
$$

and minimax reconstruction [12] corresponds to the choice

$$
H_{\mathrm{MX0}}\left(e^{j 2 \pi f}\right)=\frac{\sum_{k \in \mathbb{Z}} \Phi^{*}(f+k) \widetilde{\Phi}(f+k)}{\sum_{k \in \mathbb{Z}}|\Phi(f+k)|^{2} \sum_{k \in \mathbb{Z}}|\widetilde{\Phi}(f+k)|^{2}} .
$$

Note that the filter (4) is not defined when the denominator is zero; it can be shown that if $\varphi(t)$ and $\tilde{\varphi}(t)$ are Riesz generators (see the next section) and $\mathcal{V} \oplus \mathcal{V}^{\perp}=L_{2}$, where $\oplus$ denotes the direct sum, then the denominator is bounded away from zero [27, Proposition 4.8]. The strategies we propose in the noisy case generalize these two solutions: the least-squares methods generalize (4), while the minimax MSE and Wiener approaches lead to an extension of (5).

\section{A. Mathematical Hypotheses}

The reconstruction scheme of Fig. 3 establishes a one-to-one correspondence between the number of known parameters (measurements) and unknowns (i.e., the coefficients $c[n]$ of $\hat{x}(t)$ in (2)). However, this does not yet mean that the problem is well posed mathematically. We still need to introduce a few safeguards and definitions.

First, we need to make sure that the samples of the noisefree signal $y_{0}[n]=\langle\tilde{\varphi}(t-n), x(t)\rangle$ are well defined. In the deterministic case, we therefore specify our class of admissible input signals $x(t)$ as

$$
\mathcal{H}=\left\{x(t): \int_{-\infty}^{+\infty}|\widetilde{\Phi}(f)|^{2}|X(f)|^{2}\left(1+|2 \pi f|^{2}\right) d f<+\infty\right\}
$$

where $X(f)$ is the Fourier transform of $x(t)$. In other words, we are imposing that the continuous-time signal that is fed into the ideal sampler is at least differentiable once in the $L_{2}$-sense, which implies pointwise continuity. This ensures that $y_{0}[n] \in \ell_{2}$ (cf. [28], App. C) and allows us to express the discrete-time Fourier transform of $y_{0}[n]$ as

$$
Y_{0}\left(e^{j 2 \pi f}\right)=\sum_{k \in \mathbb{Z}} \widetilde{\Phi}^{*}(f+k) X(f+k) .
$$

In the stochastic case, we have a similar admissibility condition where $|X(f)|^{2}$ in (6) is replaced by $S_{x}(f)$, the spectral power density of $x(t)$.

The second requirement is that the discrete/continuous representation of the solution (2) be stable and unambiguous. Specifically, we impose that the functions $\{\varphi(t-n)\}_{n \in \mathbb{Z}}$ form a Riesz basis of $\mathcal{V}$ [13], which ensures that the representation is unique and that there is a direct equivalence between the discrete-time and continuous-time quadratic norms of the solution: $\|c\|_{\ell_{2}}^{2}$ and $\|\hat{x}\|_{L_{2}}^{2}$, respectively. The Riesz basis condition is equivalent to the requirement that there exist two constants $0<C_{1}$ and $C_{2}<+\infty$ (the lower and upper Riesz bounds) such that

$$
C_{1}<A_{\varphi}\left(e^{j 2 \pi f}\right)<C_{2}
$$

almost everywhere, where

$$
A_{\varphi}\left(e^{j 2 \pi f}\right)=\sum_{k \in \mathbb{Z}}|\Phi(f+k)|^{2}
$$

is the Fourier transform of the Gram sequence $a_{\varphi}[n]=$ $\langle\varphi(t), \varphi(t+n)\rangle$ (cf. [13]). This also means that $A_{\varphi}\left(e^{j 2 \pi f}\right)$ cannot vanish anywhere if it is a continuous function of $f$, which is precisely the case here because of (10) below.

Since the noisy samples $y[n]$ specified by (1) are not necessarily in $\ell_{2}$, we want a slightly stronger $L_{p}$ stability condition for all $1 \leq p \leq+\infty$, which can be enforced by the additional requirement (cf. [14])

$$
\sup _{t \in[0,1]} \sum_{n \in \mathbb{Z}}|\varphi(t-n)|<+\infty .
$$

This condition implies that the continuous-time reconstruction $\hat{x}(t)$ will be bounded whenever its coefficients $c[n]$ are bounded, and vice versa. Globally, this ensures a BIBO-i.e., bounded (discrete) input, bounded (continuous) output-behavior whenever the digital correction filter $h[n]$ in Fig. 3 is stable (i.e., $h \in \ell_{1}$ ).

For convenience, we summarize the assumptions made throughout the paper on the input signal and the sampling and reconstruction filters.

- Input signal $x(t)$ and sampling filter $\tilde{\varphi}(-t)$ satisfy (6);

- Reconstruction filter $\varphi(t)$ satisfies (10) and $0<$ $A_{\varphi}\left(e^{j 2 \pi f}\right)<+\infty$, where $A_{\varphi}\left(e^{j 2 \pi f}\right)$, is defined by (9). 


\section{B. Basic Tools for Energy Calculations}

In our derivations of the filter $h[n]$, we rely on the following two useful identities. The first is Parseval's theorem, which states that for any two sequences $d[n] \in \ell_{2}$ and $g[n] \in \ell_{2}$,

$$
\langle d, g\rangle_{\ell_{2}}=\sum_{n \in \mathbb{Z}} d[n] g[n]=\int_{0}^{1} D\left(e^{j 2 \pi f}\right) G^{*}\left(e^{j 2 \pi f}\right) d f
$$

where $D\left(e^{j 2 \pi f}\right)$ and $G\left(e^{j 2 \pi f}\right)$ are the discrete-time Fourier transforms of $d[n]$ and $g[n]$, respectively. The second follows from the Poisson sum formula

$$
\sum_{n \in \mathbb{Z}} z(n) e^{-j 2 \pi f n}=\sum_{k \in \mathbb{Z}} Z(f+k)
$$

where $z(n)=\left.z(t)\right|_{t=n}$, and $Z(f)=\int_{-\infty}^{+\infty} z(t) e^{-j 2 \pi f t} d t$ is the Fourier transform of $z(t)$. The classical hypotheses for (12) to converge uniformly are: i) $Z(f) \in L_{1}$ and ii) $z(n) \in \ell_{1}$ [29]. In the slightly weaker $l_{2}$ setting, the relation is true almost everywhere. Also, because we will be mostly integrating positive terms, we will be able to freely interchange the order of summation and integration (Fubini's theorem). For instance, if $G(f) \geq 0$ and the left-hand integral is well defined, then we can safely write

$$
\int_{-\infty}^{+\infty} G(f) d f=\sum_{k \in \mathbb{Z}} \int_{0}^{1} G(f+k) d f=\int_{0}^{1} \sum_{k \in \mathbb{Z}} G(f+k) d f .
$$

\section{RECONSTRUCTION FILTERS}

We now investigate several different design criteria for $h[n]$ in Fig. 3 and derive the corresponding solutions. We begin with the deterministic least-squares approach in Section III-A, which results in an inverse filter. We then move on to regularization methods, which are better suited for solving inverse problems in the presence of noise [30]. Specifically, we apply the Tikhonov-Phillips technique, which imposes a smoothness constraint on the signal; this leads to the derivation of a regularized least-squares, or Tikhonov, filter in Section III-B. Departing partially from the deterministic setting, in Section III-C, we assume a random noise process and consider minimax MSE strategies. Finally, in Section III-D, we adopt a Wiener-type formulation where both the signal and noise are treated as realizations of stationary processes and minimize an appropriate MSE measure.

\section{A. Least Squares}

The most straightforward design strategy is based on a leastsquares criterion

$$
\epsilon_{\mathrm{LS}}=\sum_{n \in \mathbb{Z}}(\hat{y}[n]-y[n])^{2}
$$

where $y[n]$ is the noisy data and $\hat{y}[n]$ represents the samples derived from the reconstructed signal $\hat{x}(t)$ :

$$
\hat{y}[n]=\langle\tilde{\varphi}(t-n), \hat{x}(t)\rangle .
$$

In this approach, the filter $h[n]$ is selected such that the estimated samples $\hat{y}[n]$ are as close as possible to the given samples $y[n]$ in an $\ell_{2}$-norm sense.

Using Parseval's theorem, (14) can equivalently be written as

$$
\epsilon_{\mathrm{LS}}=\int_{0}^{1}\left|\widehat{Y}\left(e^{j 2 \pi f}\right)-Y\left(e^{j 2 \pi f}\right)\right|^{2} d f
$$

where $Y\left(e^{j 2 \pi f}\right)$ and $\widehat{Y}\left(e^{j 2 \pi f}\right)$ are the discrete-time Fourier transforms of $y[n]$ and $\hat{y}[n]$, respectively. More generally, introducing a positive frequency weighting kernel $S_{w}\left(e^{j 2 \pi f}\right)$ leads to the weighted least-squares criterion

$$
\epsilon_{\mathrm{LS}}=\int_{0}^{1} S_{w}^{-1}\left(e^{j 2 \pi f}\right)\left|\hat{Y}\left(e^{j 2 \pi f}\right)-Y\left(e^{j 2 \pi f}\right)\right|^{2} d f .
$$

To develop a solution to (17) we first note that

$$
\begin{aligned}
\widehat{Y}\left(e^{j 2 \pi f}\right) & =\sum_{k \in \mathbb{Z}} \widetilde{\Phi}^{*}(f+k) \widehat{X}(f+k) \\
& =H\left(e^{j 2 \pi f}\right) Y\left(e^{j 2 \pi f}\right) A_{\tilde{\varphi} \varphi}\left(e^{j 2 \pi f}\right)
\end{aligned}
$$

where $H\left(e^{j 2 \pi f}\right)$ is the frequency response of the filter $h[n]$ and where we defined

$$
A_{\tilde{\varphi} \varphi}\left(e^{j 2 \pi f}\right)=\sum_{k \in \mathbb{Z}} \widetilde{\Phi}(f+k) \Phi^{*}(f+k) .
$$

Here, our implicit assumption is that $\hat{y}[n] \in \ell_{2}$; this is guaranteed whenever $h[n] \in \ell_{1}$ and $\widetilde{\Phi}(f)$ is bounded.

Clearly, $\epsilon_{\mathrm{LS}}$ is minimized if we can choose $H\left(e^{j 2 \pi f}\right)$ such that $\widehat{Y}\left(e^{j 2 \pi f}\right)=Y\left(e^{j 2 \pi f}\right)$ for all $f \in \Omega$, where $\Omega$ denotes the set of frequencies $f \in[-1 / 2,1 / 2]$ such that $Y\left(e^{j 2 \pi f}\right) A_{\tilde{\varphi} \varphi}\left(e^{j 2 \pi f}\right) \neq 0$. This can be achieved with

$$
H_{\mathrm{LS}}\left(e^{j 2 \pi f}\right)=\frac{1}{A_{\tilde{\varphi} \varphi}\left(e^{j 2 \pi f}\right)}, \quad f \in \Omega
$$

For $f \notin \Omega$, the filter $H_{\mathrm{LS}}\left(e^{j 2 \pi f}\right)$ can be chosen arbitrarily. Interestingly the least-squares filter $H_{\mathrm{LS}}\left(e^{j 2 \pi f}\right)$ does not depend on the frequency weighting kernel.

The least-squares solution (20) is equivalent to the consistent reconstruction filter (4) of [8], so that in effect, this approach does not take the noise into account. The results in [8] imply that for high signal-to-noise ratio (SNR) values, and assuming $\mathcal{V} \oplus \widetilde{\mathcal{V}}^{\perp}=L_{2}$, the reconstructed signal $\hat{x}_{\mathrm{LS}}(t)$ approaches the oblique projection $E_{\mathcal{V} \widetilde{\mathcal{V}} \perp} x(t)$ onto $\mathcal{V}$ along $\widetilde{\mathcal{V}}^{\perp}$, which is the unique operator satisfying

$$
\begin{aligned}
& E_{\mathcal{\mathcal { V }}^{\perp}} v(t)=v(t), \quad \forall v(t) \in \mathcal{V} \\
& E_{\mathcal{V}^{\perp}{ }^{\perp}} \tilde{v}(t)=0, \quad \forall \tilde{v}(t) \in \widetilde{\mathcal{V}} .
\end{aligned}
$$


The drawback of the least-squares approach is that if $A_{\tilde{\varphi} \varphi}\left(e^{j 2 \pi f}\right)$ is close to zero for some frequency $f$, then $H_{\mathrm{LS}}\left(e^{j 2 \pi f}\right)$ will be large at that frequency, leading to noise enhancement. This property of the least-squares solution is demonstrated in the context of concrete examples in Section V. Furthermore, the least-squares strategy is aimed at minimizing the difference between $y[n]$ and $\hat{y}[n]$ but does not guarantee a small difference between $\hat{x}(t)$ and $x(t)$.

\section{B. Regularized Least Squares}

To improve the performance of the least-squares filter, a popular modification is the regularized least-squares, or Tikhonov, approach in which a regularization term is added to the data fitting error (17):

$$
\begin{aligned}
\epsilon_{\mathrm{TIK}}=\int_{0}^{1} S_{w}^{-1}\left(e^{j 2 \pi f}\right)\left|\widehat{Y}\left(e^{j 2 \pi f}\right)-Y\left(e^{j 2 \pi f}\right)\right|^{2} d f & \\
& +\lambda \int_{-\infty}^{+\infty}|L(f)|^{2}|\widehat{X}(f)|^{2} d f
\end{aligned}
$$

for some frequency weighting function $|L(f)|^{2}>0$ and scalar $\lambda \geq 0$, where $\widehat{Y}\left(e^{j 2 \pi f}\right)$ is given by (18) and

$$
\widehat{X}(f)=H\left(e^{j 2 \pi f}\right) Y\left(e^{j 2 \pi f}\right) \Phi(f) .
$$

The error measure $\epsilon_{\text {TIK }}$ can be viewed as the Lagrangian associated with the problem of minimizing $\epsilon_{\mathrm{LS}}$ of (17) subject to the constraint that the reconstructed signal $\hat{x}(t)$ lies in the class $\mathcal{G}$ defined by

$$
\mathcal{G}=\left\{x(t): \int_{-\infty}^{+\infty}|L(f)|^{2}|X(f)|^{2} d f \leq \sigma_{0}^{2}\right\} .
$$

By introducing the regularization operator $\mathrm{L}$ whose frequency response is $L(f)$, this condition can be expressed compactly as $\|\mathrm{L} x\|_{L_{2}} \leq \sigma_{0}$. In this case, it follows from the Karush-Kuhn-Tucker conditions [31] that at the optimal solution

$$
\lambda \cdot\left(\|\mathrm{L} \hat{x}\|_{L_{2}}^{2}-\sigma_{0}^{2}\right)=0
$$

so that either the equality constraint is satisfied for $\widehat{X}(f)$, or $\lambda=0$.

The derivation of the Tikhonov filter is relegated to Appendix I in which we show that

$$
\begin{aligned}
& H_{\mathrm{TIK}}\left(e^{j 2 \pi f}\right) \\
& =\frac{A_{\tilde{\varphi} \varphi}\left(e^{j 2 \pi f}\right)}{\left|A_{\tilde{\varphi} \varphi}\left(e^{j 2 \pi f}\right)\right|^{2}+\lambda S_{w}\left(e^{j 2 \pi f}\right) \sum_{k \in \mathbb{Z}}|L(f+k)|^{2}|\Phi(f+k)|^{2}}
\end{aligned}
$$

for frequencies $f$ for which $Y\left(e^{j 2 \pi f}\right) \neq 0$, where $A_{\tilde{\varphi} \varphi}\left(e^{j 2 \pi f}\right)$ is defined by (19). The solution can be obtained by imposing the condition that all Gateaux differentials are zero, where we assume that $\sum_{k \in \mathbb{Z}}|L(f+k)|^{2}|\Phi(f+k)|^{2}<+\infty$. Although $H_{\mathrm{TIK}}\left(e^{j 2 \pi f}\right)$ is arbitrary on values of $f$ for which $Y\left(e^{j 2 \pi f}\right)=$ 0 , the choice of $H_{\mathrm{TIK}}\left(e^{j 2 \pi f}\right)$ on these frequencies will not affect the reconstructed output $\hat{x}(t)$. For $\lambda=0$, the Tikhonov filter (27) reduces to the least-squares filter (20). If $\lambda>0$, then $\left|H_{\mathrm{TIK}}\left(e^{j 2 \pi f}\right)\right|<\left|H_{\mathrm{LS}}\left(e^{j 2 \pi f}\right)\right|$, since $|L(f)|^{2}>0$ and $|\Phi(f)|^{2}>0$ for some $f$ because of (8).

In the inequality-constrained version of the Tikhonov filter $\lambda$ must be chosen to satisfy (26). Denoting by $\hat{x}_{\mathrm{TIK}, \lambda}$ the reconstructed signal resulting from the Tikhonov filter (27) for a fixed value of $\lambda$, this implies the following procedure for selecting $\lambda$ : if $\left\|\mathrm{L} \hat{x}_{\mathrm{TIK}, 0}\right\|_{L_{2}} \leq \sigma_{0}$, then $\lambda=0$. Otherwise, $\lambda>0$ is a parameter that depends on the data $y[n]$ and is chosen such that $\left\|\mathrm{L} \hat{x}_{\mathrm{TIK}, \lambda}\right\|_{L_{2}}=\sigma_{0}$. In this case $\lambda$ does not have an explicit formula, but, rather, is determined as the solution of a data-dependent, nonlinear equation.

\section{Minimax MSE Filters}

Both the least-squares and the regularized least-squares (Tikhonov) algorithms are based on minimizing a data-error criterion; i.e., a criterion that depends on the error between $y[n]$ and $\hat{y}[n]$ where $\hat{y}[n]=\langle\tilde{\varphi}(t-n), \hat{x}(t)\rangle$. However, in an estimation context, we typically would like to minimize the estimation error $x(t)-\hat{x}(t)$. A popular measure of this error is the MSE $\mathrm{E}\left\{|\hat{x}(t)-x(t)|^{2}\right\}$, which is the average squared-norm estimation error. Computing the MSE in our setting in which the signal $x(t)$ is deterministic shows that it depends explicitly on $x(t)$, and therefore cannot be minimized. (This is in contrast with the stochastic setting in which $x(t)$ is a stationary random process, as we discuss in Section III-D.) Assuming, as in the previous section, that $x(t)$ belongs to the class $\mathcal{G}$ defined by (25), we can obtain a signal-independent error measure by considering the worst-case MSE on $\mathcal{G}$. The generic case in which the only information we have is that $x(t) \in L_{2}$ can be treated in this framework by choosing $L(f)=1$ and $\sigma_{0}^{2} \rightarrow+\infty$.

Having combated the signal dependence of the MSE, we are now faced with another problem (which remains also in the stochastic case): The filter minimizing the worst-case MSE depends on the time index $t=t_{0}$ so that in principle, a different filter $h[n]$ is optimal for each $t_{0}$. To obtain a fixed solution $h[n]$ for all $t_{0}$, we develop two strategies. The first approach is inspired by some of our previous work [12] and is based on the observation that, even in the absence of noise, we cannot in general have $\hat{x}(t)=x(t)$ because the reconstruction is constrained to lie in the space $\mathcal{V}$. Since the minimal norm approximation to $x(t)$ in $\mathcal{V}$ is $x \mathcal{V}(t)=P_{\mathcal{V}} x(t)$, instead of treating the error $\hat{x}(t)-x(t)$, we consider the projected error $\hat{x}(t)-x_{\mathcal{V}}(t)$ at a particular time instance $t_{0}$ and seek the filter $h[n]$ that minimizes the worst-case projected MSE over $\mathcal{G}$

$$
\epsilon_{\mathrm{PROJ}}=\max _{x(t) \in \mathcal{G}} \mathrm{E}\left\{\left|\hat{x}\left(t_{0}\right)-x_{\mathcal{V}}\left(t_{0}\right)\right|^{2}\right\} .
$$

Fortunately, the resulting filter does not depend on $t_{0}$; therefore, the same filtering algorithm provides the optimal solution for 
each $t_{0}$. In the second approach, the filter is designed to minimize the time-average worst-case MSE

$$
\epsilon_{\mathrm{AVG}}=\lim _{T \rightarrow+\infty} \frac{1}{2 T} \int_{-T}^{T} \max _{x(t) \in \mathcal{G}} \mathrm{E}\left\{\left|\hat{x}\left(t_{0}\right)-x\left(t_{0}\right)\right|^{2}\right\} d t_{0} .
$$

Interestingly, we will see that these two strategies lead to the same reconstruction filter.

1) Minimax Projected MSE: We begin by choosing the filter to minimize the worst-case projected MSE over $x(t) \in \mathcal{G}$, so that $h[n]$ is the solution to

$$
\min _{h[n]} \max _{x(t) \in \mathcal{G}} \mathrm{E}\left\{\left|\hat{x}\left(t_{0}\right)-x_{\mathcal{V}}\left(t_{0}\right)\right|^{2}\right\} .
$$

The development of $h[n]$ is more conveniently carried out in the frequency domain. The following lemmas will enable us to obtain a Fourier-domain formulation of (30).

Lemma 1: Let $\hat{x}(t)$ be the signal reconstruction defined by (1) and (2), where $w[n]$ is a zero-mean stationary noise process with power spectrum $S_{w}\left(e^{j 2 \pi f}\right)$ and let $x_{\mathcal{V}}(t)=P_{\mathcal{V}} x(t)$. Then

$$
\begin{gathered}
\mathrm{E}\left\{\left|\hat{x}\left(t_{0}\right)-x_{\mathcal{V}}\left(t_{0}\right)\right|^{2}\right\}=\left|\int_{0}^{1} \sum_{k \in \mathbb{Z}} X(f+k) Z_{t_{0}}(f+k) d f\right|^{2} \\
+\int_{0}^{1}\left|H\left(e^{j 2 \pi f}\right)\right|^{2} S_{w}\left(e^{j 2 \pi f}\right)\left|B_{t_{0}}\left(e^{j 2 \pi f}\right)\right|^{2} d f
\end{gathered}
$$

where

$$
B_{t_{0}}\left(e^{j 2 \pi f}\right)=\sum_{\ell \in \mathbb{Z}} \Phi(f+\ell) e^{j 2 \pi(f+\ell) t_{0}}
$$

and

$$
Z_{t_{0}}(f)=B_{t_{0}}\left(e^{j 2 \pi f}\right)\left(\widetilde{\Phi}^{*}(f) H\left(e^{j 2 \pi f}\right)-\frac{1}{A_{\varphi}\left(e^{j 2 \pi f}\right)} \Phi^{*}(f)\right) .
$$

Proof: See Appendix II.

Lemma 2: Let

$$
\mathcal{G}=\left\{x(t): \int_{-\infty}^{+\infty}|L(f)|^{2}|X(f)|^{2} d f \leq \sigma_{0}^{2}\right\}
$$

for some $L(f)>0$. Then, for any function $Z(f)$ such that

$$
\int_{-\infty}^{+\infty}|Z(f)|^{2} /|L(f)|^{2} d f<+\infty
$$

we have

$$
\begin{aligned}
\max _{x(t) \in \mathcal{G}} \mid \int_{0}^{1} \sum_{k \in \mathbb{Z}} X(f & +k)\left.Z(f+k) d f\right|^{2} \\
& =\sigma_{0}^{2} \int_{0}^{1} \sum_{k \in \mathbb{Z}}|Z(f+k)|^{2} /|L(f+k)|^{2} d f .
\end{aligned}
$$

Proof: See Appendix III.
Using Lemmas 1 and 2, and assuming that $\sum_{k \in \mathbb{Z}} \mid \widetilde{\Phi}(f+$ $k)\left.\right|^{2} /|L(f+k)|^{2}<\infty, \sum_{k \in \mathbb{Z}}|\Phi(f+k)|^{2} /|L(f+k)|^{2}<\infty$, the problem (30) can be expressed in the Fourier domain as

$$
\min _{H\left(e^{j 2 \pi f}\right)} \int_{0}^{1} D\left(e^{j 2 \pi f}\right)\left|B_{t_{0}}\left(e^{j 2 \pi f}\right)\right|^{2} d f
$$

where

$$
\begin{aligned}
& D\left(e^{j 2 \pi f}\right)=\left|H\left(e^{j 2 \pi f}\right)\right|^{2} S_{w}\left(e^{j 2 \pi f}\right)+\sigma_{0}^{2} \\
& \cdot \sum_{k \in \mathbb{Z}}\left|\widetilde{\Phi}^{*}(f+k) H\left(e^{j 2 \pi f}\right)-\frac{\Phi^{*}(f+k)}{A_{\varphi}\left(e^{j 2 \pi f}\right)}\right|^{2} /|L(f+k)|^{2} .
\end{aligned}
$$

Since $D\left(e^{j 2 \pi f}\right) \geq 0$, the minimax filter can be obtained by minimizing the convex function $D\left(e^{j 2 \pi f}\right)$, which does not depend on $t_{0}$. Setting the derivative to zero results in the minimax MSE filter

$$
\begin{aligned}
& H_{\mathrm{MX}}\left(e^{j 2 \pi f}\right) \\
& A_{\varphi}\left(e^{j 2 \pi f}\right)\left(S_{w}\left(e^{j 2 \pi f}\right)+\sigma_{0}^{2} \sum_{k \in \mathbb{Z}}|\widetilde{\Phi}(f+k)|^{2} /|L(f+k)|^{2}\right)
\end{aligned}
$$

It is interesting to evaluate the minimax filter when the only prior information on $x(t)$ is that $x(t) \in L_{2}$. Substituting $L(f)=$ 1 and $\sigma_{0}^{2} \rightarrow+\infty$ into (36) yields

$$
H_{\mathrm{MX} 0}\left(e^{j 2 \pi f}\right)=\frac{\sum_{k \in \mathbb{Z}} \Phi^{*}(f+k) \widetilde{\Phi}(f+k)}{\sum_{k \in \mathbb{Z}}|\Phi(f+k)|^{2} \sum_{k \in \mathbb{Z}}|\widetilde{\Phi}(f+k)|^{2}}
$$

which is equal to the filter (5) proposed in [12] for the noise-free case. Applying the Cauchy-Schwarz inequality to the denominator, $\left|H_{\mathrm{MX} 0}\left(e^{j 2 \pi f}\right)\right| \leq\left|H_{\mathrm{LS}}\left(e^{j 2 \pi f}\right)\right|=\left|H_{\mathrm{TIK}, 0}\left(e^{j 2 \pi f}\right)\right|$, where the last equality follows from the fact that, in this case, the Tikhonov filter and the least-squares filter coincide.

The minimax filter (37) is also obtained in the noise-free case in which $S_{w}\left(e^{j 2 \pi f}\right)=0$. From the discussion in [12], the corresponding reconstruction is $\hat{x}(t)=P_{\mathcal{V}} P_{\widetilde{\mathcal{V}}} x(t)$. Since in this case, $\mathrm{E}\left\{\left|\hat{x}\left(t_{0}\right)-x_{\mathcal{V}}\left(t_{0}\right)\right|^{2}\right\}=\left|\hat{x}\left(t_{0}\right)-x_{\mathcal{V}}\left(t_{0}\right)\right|^{2}$, we have that for every $t_{0}$

$$
\left(P_{\mathcal{V}} P_{\widetilde{\mathcal{V}}} x\right)\left(t_{0}\right)=\arg \min _{\hat{x}(t) \in \mathcal{V}} \max _{\int_{-\infty}^{+\infty}}\left|\hat{x^{2}(t) d t \leq \sigma_{0}^{2}}\right| \hat{x}\left(t_{0}\right)-\left.x \mathcal{V}\left(t_{0}\right)\right|^{2} .
$$

Therefore, in the absence of noise, besides minimizing the worst-case error energy $\int_{-\infty}^{+\infty}\left|\hat{x}(t)-x_{\mathcal{V}}(t)\right|^{2} d t$ (as established previously in [12]), $\hat{x}(t)=P_{\mathcal{V}} P_{\widetilde{\mathcal{V}}} x(t)$ also minimizes the worst-case point-wise error.

2) Average Minimax MSE: We now treat the average worstcase MSE criterion, as follows:

$$
\min _{h[n]} \lim _{T \rightarrow+\infty} \frac{1}{2 T} \int_{-T}^{T} \max _{x(t) \in \mathcal{G}} \mathrm{E}\left\{\left|\hat{x}\left(t_{0}\right)-x\left(t_{0}\right)\right|^{2}\right\} d t_{0} .
$$

Following similar steps as in the proof of Lemma 1, the MSE $\mathrm{E}\left\{\left|\hat{x}\left(t_{0}\right)-x\left(t_{0}\right)\right|^{2}\right\}$ can be expressed in the same functional form as (31), with $Z_{t_{0}}(f)$ of (33) replaced by

$$
Z_{t_{0}}(f)=\widetilde{\Phi}^{*}(f) H\left(e^{j 2 \pi f}\right) B_{t_{0}}\left(e^{j 2 \pi f}\right)-e^{j 2 \pi f t_{0}} .
$$


From Lemma 2, the maximal value of $\mathrm{E}\left\{\left|\hat{x}\left(t_{0}\right)-x\left(t_{0}\right)\right|^{2}\right\}$ over $x(t) \in \mathcal{G}$, denoted $M\left(t_{0}\right)$, is then

$$
\begin{aligned}
M\left(t_{0}\right)=\int_{0}^{1} & \left(\left|H\left(e^{j 2 \pi f}\right)\right|^{2} S_{w}\left(e^{j 2 \pi f}\right)\left|B_{t_{0}}\left(e^{j 2 \pi f}\right)\right|^{2}\right. \\
& \left.+\sigma_{0}^{2} \sum_{k \in \mathbb{Z}}\left|Z_{t_{0}}(f+k)\right|^{2} /|L(f+k)|^{2}\right) d f .
\end{aligned}
$$

Since the dependence of $M\left(t_{0}\right)$ on $t_{0}$ is only through the terms $\left|B_{t_{0}}\left(e^{j 2 \pi f}\right)\right|^{2}$ and $\left|Z_{t_{0}}(f+k)\right|^{2}$, which are both periodic in $t_{0}$ with period 1 , it follows that $M\left(t_{0}\right)$ is also 1-periodic. Therefore

$\lim _{T \rightarrow+\infty} \frac{1}{2 T} \int_{-T}^{T} \max _{x(t) \in \mathcal{G}} \mathrm{E}\left\{\left|\hat{x}\left(t_{0}\right)-x\left(t_{0}\right)\right|^{2}\right\} d t_{0}=\int_{0}^{1} M\left(t_{0}\right) d t_{0}$

and our problem becomes

$$
\min _{h[n]} \int_{0}^{1} M\left(t_{0}\right) d t_{0}
$$

In Appendix IV, we show that the solution to (43) is the same as for the previous problem and is given by (36).

We summarize our results on minimax MSE reconstruction in the following theorem.

Theorem 1: Let $y[n]=\langle\tilde{\varphi}(t-n), x(t)\rangle+w[n]$ denote noisy samples of an unknown deterministic signal $x(t)$, where $\tilde{\varphi}(t)$ is a known sampling function and $w[n]$ is a zero-mean stationary noise process with power spectrum $S_{w}\left(e^{j 2 \pi f}\right)$. Let $\hat{x}(t)=\sum_{n \in \mathbb{Z}}(h * y)[n] \varphi(t-n)$ denote a reconstruction of $x(t)$ where $\varphi(t)$ is a given reconstruction function and $h[n]$ is a discrete-time filter, let $\mathcal{G}=\left\{x(t): \int_{-\infty}^{+\infty}|L(f)|^{2}|X(f)|^{2} d f \leq \sigma_{0}^{2}\right\}$ for some $|L(f)|>0$ and $\sigma_{0}^{2}>0$, and let $x_{\mathcal{V}}(t)=P_{\mathcal{V}} x(t)$. Then the following minimax problems result in the same optimal filter $h[n]$ :

- $\min _{h[n]} \max _{x(t) \in \mathcal{G}} \mathrm{E}\left\{\left|\hat{x}\left(t_{0}\right)-x_{\mathcal{V}}\left(t_{0}\right)\right|^{2}\right\}$;

- $\min _{h[n]} \lim _{T \rightarrow+\infty}(1 / 2 T) \int_{-T}^{T} \max _{x(t) \in \mathcal{G}} \mathrm{E}\left\{\mid \hat{x}\left(t_{0}\right)-\right.$ $\left.\left.x\left(t_{0}\right)\right|^{2}\right\} d t_{0}$.

The frequency response of the filter is independent of $t_{0}$ and is given by the equation shown at bottom of page, where $\widetilde{\Phi}(f)$ and $\Phi(f)$ are the Fourier transforms of $\tilde{\varphi}(t)$ and $\varphi(t)$, respectively. In addition, if $w[n]=0$ and $L(f)=1$, then $\hat{x}(t)=P_{\mathcal{V}} P_{\widetilde{\mathcal{V}}} x(t)$.

\section{Continuous-Discrete Wiener Filter}

We now consider a full stochastic setting, where $x(t)$ is a realization of a continuous-time zero-mean stationary random process with power spectral density $S_{x}(f)$, and $w[n]$ is a zeromean stationary noise process with power spectrum $S_{w}\left(e^{j 2 \pi f}\right)$, independent of $x(t)$. Since $x(t)$ is now random, the MSE averages the squared-norm also over the signal, leading to a signalindependent expression. In principle, therefore, the filter $h[n]$ can be designed to directly minimize the MSE. Unfortunately, as in the deterministic signal case, the resulting filter depends on the time index $t$. If instead we minimize the projected MSE $\mathrm{E}\left\{\left|\hat{x}\left(t_{0}\right)-x_{\mathcal{V}}\left(t_{0}\right)\right|^{2}\right\}$, then the optimal solution is independent of time, as incorporated in the following theorem.

Theorem 2: Consider the setup of Theorem 1 where now $x(t)$ is zero-mean stationary random process with power spectral density $S_{x}(f) \in L_{2}$, independent of $w[n]$. Then, the filter minimizing the projected MSE $\mathrm{E}\left\{\left|\hat{x}\left(t_{0}\right)-x_{\mathcal{V}}\left(t_{0}\right)\right|^{2}\right\}$ is independent of $t_{0}$ and is given in the Fourier domain by $H_{\mathrm{W}}\left(e^{j 2 \pi f}\right)$

$$
=\frac{\sum_{k \in \mathbb{Z}} S_{x}(f+k) \Phi^{*}(f+k) \widetilde{\Phi}(f+k)}{A_{\varphi}\left(e^{j 2 \pi f}\right)\left(S_{w}\left(e^{j 2 \pi f}\right)+\sum_{k \in \mathbb{Z}} S_{x}(f+k)|\widetilde{\Phi}(f+k)|^{2}\right)} .
$$

Proof: See Appendix V.

Comparing the Wiener filter (44) with the minimax filter of Theorem 1, we see that the two filters have a similar form, with $\sigma_{0}^{2} /|L(f)|^{2}$ in the minimax filter replaced by the power spectrum $S_{x}(f)$ in the Wiener filter. Thus, we can view the minimax filter as a Wiener filter matched to a power spectrum $S_{x}(f)=\sigma_{0}^{2} /|L(f)|^{2}$.

\section{COMPARISON BETwEen THE DIFFERENT FILTERS}

The filtering algorithms proposed in the previous section were derived based on the minimization of a suitable cost function. The suggested methods differ in the assumptions that have been made and are summarized in Table I. The second formula in each of the entries gives the $z$-transform of the various filters which can be determined explicitly when the analysis filter has a rational transfer function and the synthesis function is compactly supported (cf. Section V).

We have seen already that the Wiener and minimax MSE filters have a similar structure, independent of the choice of $\tilde{\varphi}(t)$ and $\varphi(t)$. In Section IV-A, we consider bandlimited interpolation and show that in this case the Tikhonov filter also shares this form. When $\varphi(t)=\tilde{\varphi}(t)$, we establish the MSE superiority of the minimax MSE filter by showing that the MSE of the minimax reconstruction is smaller than that of the least-squares reconstruction for all $x(t) \in \mathcal{G}$ (not only the worst-case $x(t)$ ).

\section{A. Bandlimited Reconstruction}

Suppose that we interpolate the given samples $y[n]$ to a bandlimited function by choosing $\varphi(t)=\operatorname{sinc}(t)$. It is tempting in this case to replace the continuous-discrete model of Fig. 3 by the discrete-time model depicted in Fig. 4 and define the different error measures directly on the discrete representation in which $x[n]=\left.x(t)\right|_{t=n}$ and $f[n]=(\tilde{\varphi} * \varphi)(n)$ are the samples at times $t=n$ of the bandlimited version of $\tilde{\varphi}(t)$. As we now show, this equivalence holds under the least-squares and

$$
H_{\mathrm{MX}}\left(e^{j 2 \pi f}\right)=\frac{\sigma_{0}^{2} \sum_{k \in \mathbb{Z}} \Phi^{*}(f+k) \widetilde{\Phi}(f+k) /|L(f+k)|^{2}}{\sum_{k \in \mathbb{Z}}|\Phi(f+k)|^{2}\left(S_{w}\left(e^{j 2 \pi f}\right)+\sigma_{0}^{2} \sum_{k \in \mathbb{Z}}|\widetilde{\Phi}(f+k)|^{2} /|L(f+k)|^{2}\right)} .
$$


TABLE I

COMPARISON OF METHODS FOR SIGNAL RECOVERY

\begin{tabular}{|c|c|c|c|c|}
\hline & Signal model & Noise model & $\begin{array}{l}\text { Criterion to be } \\
\text { minimized }\end{array}$ & Formula \\
\hline Least-squares filter & no constraint & irrelevant & Data term & (20) or $(63)$ \\
\hline Tikhonov filter & $\begin{array}{l}\text { Deterministic: } \\
x \in \mathcal{G}\end{array}$ & not explicit & $\begin{array}{l}\text { Data term }+ \text { regulariza- } \\
\text { tion }\end{array}$ & (27) or (67) \\
\hline $\begin{array}{l}\text { Projected minimax fil- } \\
\text { ter }\end{array}$ & $\begin{array}{l}\text { Deterministic: } \\
x \in \mathcal{G}\end{array}$ & stationary process & $\begin{array}{l}\text { Worst-case projected } \\
\text { MSE at } t=t_{0}\end{array}$ & $(36)$ or $(68)$ \\
\hline $\begin{array}{l}\text { Average minimax } \\
\text { filter }\end{array}$ & $\begin{array}{l}\text { Deterministic: } \\
x \in \mathcal{G}\end{array}$ & stationary process & $\begin{array}{l}\text { Worst-case MSE aver- } \\
\text { aged over } t\end{array}$ & $(36)$ or $(68)$ \\
\hline Wiener filter & stationary process & stationary process & projected MSE at $t=t_{0}$ & $(44)$ or $(68)$ \\
\hline
\end{tabular}

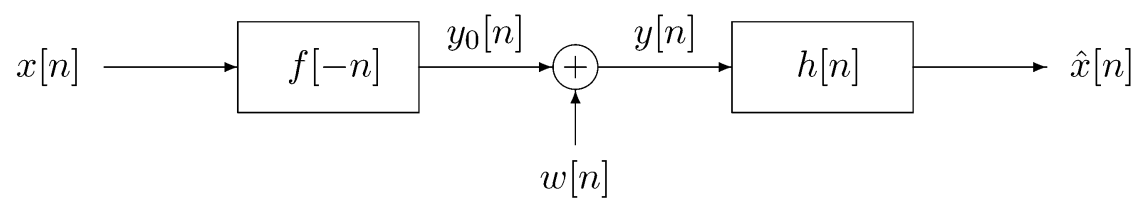

Fig. 4. Discrete-time deconvolution.

Tikhonov formulations. In the minimax MSE and Wiener approaches, an additional bandlimited constraint on the input is required.

Starting with the least-squares method, and using the fact that

$$
\Phi(f)= \begin{cases}1, & |f| \leq 1 / 2 \\ 0, & |f|>1 / 2\end{cases}
$$

the least-squares filter of (20) reduces to

$$
H_{\mathrm{LS}}\left(e^{j 2 \pi f}\right)=\frac{1}{\widetilde{\Phi}^{*}(f)}, \quad f: \widetilde{\Phi}^{*}(f) \neq 0 .
$$

Noting that the Fourier transform of $f[n]$ is $F\left(e^{j 2 \pi f}\right)=\widetilde{\Phi}(f)$ for $|f| \leq 1 / 2$, we can express the filter as $H_{\mathrm{LS}}\left(e^{j 2 \pi f}\right)=$ $1 / F^{*}\left(e^{j 2 \pi f}\right)$, which is precisely the least-squares solution to the problem of minimizing the data error in the discrete-time deconvolution problem of Fig. 4.

Similarly, under the model (45), the Tikhonov filter becomes

$$
H_{\mathrm{TIK}}\left(e^{j 2 \pi f}\right)=\frac{\tilde{\Phi}(f)}{|\widetilde{\Phi}(f)|^{2}+\lambda S_{w}\left(e^{j 2 \pi f}\right)|L(f)|^{2}}, \quad|f| \leq 1 / 2 .
$$

This solution can also be interpreted as the Tikhonov filter corresponding to the equivalent discrete-time model, subject to the constraint that $\hat{x}[n]$ lies in the class $\mathcal{G}_{d}$ defined by

$$
\mathcal{G}_{d}=\left\{x[n]: \int_{-1 / 2}^{1 / 2}\left|L_{d}\left(e^{j 2 \pi f}\right)\right|^{2}\left|X\left(e^{j 2 \pi f}\right)\right|^{2} d f \leq \sigma_{0}^{2}\right\}
$$

where $L_{d}\left(e^{j 2 \pi f}\right)$ is the discrete filter satisfying $L_{d}\left(e^{j 2 \pi f}\right)=$ $L(f)$ for $|f| \leq 1 / 2$.

Since the least-squares and Tikhonov criteria depend only on $\hat{x}(t)$, the fact that $\varphi(t)$ is bandlimited reduces the entire problem to the discrete-time version of Fig. 4. This is in contrast with the minimax MSE filter, which becomes

$$
\begin{aligned}
& H_{\mathrm{Mx}}\left(e^{j 2 \pi f}\right) \\
& =\frac{\sigma_{0}^{2} \widetilde{\Phi}(f) /|L(f)|^{2}}{S_{w}\left(e^{j 2 \pi f}\right)+\sigma_{0}^{2} \sum_{k \in \mathbb{Z}}|\widetilde{\Phi}(f+k)|^{2} /|L(f+k)|^{2}}
\end{aligned}
$$

for $|f| \leq 1 / 2$. A functionally equivalent equation is also obtained for the Wiener filter (44) with $S_{x}(f)$ playing the role of $\sigma_{0}^{2} /|L(f)|^{2}$. The minimax solution (49) does not have a corresponding discrete-time interpretation because of the infinite sum in the denominator. To convert the problem into the discrete form associated with Fig. 4, we need to add the constraint that the input $x(t)$ is bandlimited, or, equivalently, that the analysis filter is such that $\widetilde{\Phi}(f)=0$ for $|f|>1 / 2$. In this case

$$
H_{\mathrm{MX}}\left(e^{j 2 \pi f}\right)=\frac{\sigma_{0}^{2} \widetilde{\Phi}(f) /|L(f)|^{2}}{S_{w}\left(e^{j 2 \pi f}\right)+\sigma_{0}^{2}|\widetilde{\Phi}(f)|^{2} /|L(f)|^{2}},|f| \leq 1 / 2
$$

which is equal to the minimax MSE deconvolution filter that minimizes the worst-case MSE given by $\max _{x[n] \in \mathcal{G}_{d}} E\left\{\left|\hat{x}\left[n_{0}\right]-x\left[n_{0}\right]\right|^{2}\right\}[32]$.

If we choose $S_{x}(f)=\sigma_{0}^{2} /|L(f)|^{2}$ in (50), then $H_{\mathrm{MX}}\left(e^{j 2 \pi f}\right)=H_{\mathrm{W}}\left(e^{j 2 \pi f}\right)$ reduces to

$$
H_{\mathrm{W}}\left(e^{j 2 \pi f}\right)=\frac{S_{x}(f) \widetilde{\Phi}(f)}{S_{w}\left(e^{j 2 \pi f}\right)+S_{x}(f)|\widetilde{\Phi}(f)|^{2}} .
$$

The filter (51) is the classical Wiener filter for the problem of estimating a random process $x[n]$ from blurred, noisy observations.

Comparing (47), (50), and (51), we see that in the case of bandlimited interpolation, the Tikhonov, minimax, and Wiener filters are equivalent provided that $S_{x}(f)=\sigma_{0}^{2} /|L(f)|^{2}$ and $\lambda=1 / \sigma_{0}^{2}$. 


\section{B. Equal Sampling and Reconstruction Spaces}

The least-squares filter makes no prior assumption on $x(t)$. The Tikhonov and minimax MSE filters, on the other hand, assume that $x(t) \in \mathcal{G}$, where $\mathcal{G}$ is defined by (25). Since these filters incorporate further knowledge on the input $x(t)$, we expect intuitively that their performance will be superior to that of the least-squares reconstruction. We now demonstrate that this intuition is often correct, at least for the minimax MSE filter, by examining the special case in which $\tilde{\varphi}(t)=\varphi(t)$.

For $\varphi(t)=\tilde{\varphi}(t)$ the minimax MSE and least-squares filters are given, respectively, by

$$
H_{\mathrm{MX}}\left(e^{j 2 \pi f}\right)=\frac{\alpha\left(e^{j 2 \pi f}\right)}{A_{\varphi}\left(e^{j 2 \pi f}\right)}
$$

and

$$
H_{\mathrm{LS}}\left(e^{j 2 \pi f}\right)=\frac{1}{A_{\varphi}\left(e^{j 2 \pi f}\right)}
$$

where we defined

$$
\alpha\left(e^{j 2 \pi f}\right)=\frac{\sigma_{0}^{2} \sum_{k \in \mathbb{Z}}|\Phi(f+k)|^{2} /|L(f+k)|^{2}}{S_{w}\left(e^{j 2 \pi f}\right)+\sigma_{0}^{2} \sum_{k \in \mathbb{Z}}|\Phi(f+k)|^{2} /|L(f+k)|^{2}} \cdot
$$

We assume that $S_{w}\left(e^{j 2 \pi f}\right)>0$ over some measurable interval of $f$; otherwise, the filters (52) and (53) coincide. With $H\left(e^{j 2 \pi f}\right)=H_{\mathrm{LS}}\left(e^{j 2 \pi f}\right)$ of (53), the projected MSE at time $t_{0}$ is given from (31) by

$$
\begin{aligned}
\mathrm{E} & \left\{\left|\hat{x}\left(t_{0}\right)-x_{\mathcal{V}}\left(t_{0}\right)\right|^{2}\right\} \\
& =\int_{0}^{1} A_{\varphi}^{-2}\left(e^{j 2 \pi f}\right) S_{w}\left(e^{j 2 \pi f}\right)\left|B_{t_{0}}\left(e^{j 2 \pi f}\right)\right|^{2} d f \triangleq E_{\mathrm{LS}} .
\end{aligned}
$$

Note that the resulting MSE is independent of $x(t)$. On the other hand, if $H\left(e^{j 2 \pi f}\right)=H_{\mathrm{MX}}\left(e^{j 2 \pi f}\right)$ is the minimax MSE filter of (52), then the MSE of (31), which we denote by $E_{\mathrm{Mx}}$, will depend on $x(t)$. However, from Lemmas 1 and 2, we have that for any $x(t) \in \mathcal{G}$,

$$
\begin{aligned}
E_{\mathrm{MX}} \leq & \int_{0}^{1}\left|B_{t_{0}}\left(e^{j 2 \pi f}\right)\right|^{2} \\
& \times\left(\left|H_{\mathrm{MX}}\left(e^{j 2 \pi f}\right)\right|^{2} S_{w}\left(e^{j 2 \pi f}\right)\right. \\
& +\sigma_{0}^{2} \sum_{k \in \mathbb{Z}}|\Phi(f+k)|^{2} /|L(f+k)|^{2} \\
& \left.\times \mid H_{\mathrm{MX}}\left(e^{j 2 \pi f}\right)-\frac{1}{\left.\left.A_{\varphi}\left(e^{j 2 \pi f}\right)\right|^{2}\right)}\right) d f \\
= & \int_{0}^{1} A_{\varphi}^{-2}\left(e^{j 2 \pi f}\right) S_{w}\left(e^{j 2 \pi f}\right)\left|B_{t_{0}}\left(e^{j 2 \pi f}\right)\right|^{2} \alpha\left(e^{j 2 \pi f}\right) d f .
\end{aligned}
$$

Since $\alpha\left(e^{j 2 \pi f}\right) \leq 1$ with strict inequality for $S_{w}\left(e^{j 2 \pi f}\right)>0$, we conclude that $E_{\mathrm{MX}}<E_{\mathrm{LS}}$ for all $x(t) \in \mathcal{G}$, as long as
$S_{w}\left(e^{j 2 \pi f}\right)>0$ over some measurable interval of $f$. Note, that this property is not guaranteed to hold for the Tikhonov filter.

\section{Practical ISSUES}

The practical specification of the filters developed in Section III in the nonbandlimited case is delicate because of the various infinite sums that appear in the filter formulas. Depending on the rate of decay of $\Phi(f)$ and $\widetilde{\Phi}(f)$ as $f \rightarrow \infty$, these sums may be truncated to an appropriate number of terms and the frequency responses computed numerically to the required degree of precision. While this "brute force" approach may work for most of the filters, it is not elegant and may fail in some cases because of numeric instabilities. Here, we show how these difficulties can be bypassed and how the calculations can be done exactly in spline spaces. Specifically, we propose a practical procedure for determining the rational transfer functions ( $z$-transform) of the various reconstruction filters.

We concentrate on the case where the analysis filter is causal and is described by a rational transfer function that has the general form

$$
\widetilde{\Phi}^{*}(f)=\frac{\prod_{m=1}^{M}\left(j 2 \pi f-\gamma_{m}\right)}{\prod_{n=1}^{N}\left(j 2 \pi f-\alpha_{n}\right)}
$$

with $N>M$. In practice, the regularization operator is often chosen to be a first or second order derivative in order to constrain the solution to be smooth and nonoscillating [23], [33], [34]. Here, we will assume that the regularization operator is a differential operator of order $K$ of the form $\mathrm{L}=\mathrm{D}^{K}+$ $a_{1} \mathrm{D}^{K-1}+\cdots a_{K} \mathrm{I}$, where $\mathrm{D}$ denotes the derivative operator and $\mathrm{I}=\mathrm{D}^{0}$ is the identity. This means that its frequency response can be written as

$$
L(f)=\prod_{k=1}^{K}\left(j 2 \pi f-\lambda_{k}\right)
$$

where the $\lambda_{k} \mathrm{~s}$ are the characteristic roots of the operator, and depend on the coefficients $a_{k}$.

\section{A. Determination of the Transfer Functions}

To explain our computational approach, we show how to determine the rational form of the inverse filter (20) using a pole cancellation technique. To this end, we define the digital filter

$$
P(z)=\prod_{n=1}^{N} \frac{1}{1-e^{\alpha_{n}} z^{-1}}
$$

whose poles are in exact correspondence with those of (57). We then rewrite the analysis filter as

$$
\widetilde{\Phi}^{*}(f)=\tilde{B}(f) P\left(e^{j 2 \pi f}\right)
$$

where

$$
\tilde{B}(f)=\left(\prod_{n=1}^{N} \frac{1-e^{\alpha_{n}-j 2 \pi f}}{j 2 \pi f-\alpha_{n}}\right) \cdot \prod_{m=1}^{M}\left(j 2 \pi f-\gamma_{m}\right)
$$

is the Fourier transform of the exponential B-spline $\tilde{\beta}(t)=$ $\beta_{\left(\alpha_{1}, \cdots, \alpha_{N} ; \gamma_{1}, \cdots, \gamma_{M}\right)}(t)$, which is parameterized by its poles and 
zeros using the notation of [35]. The important point is that this function is compactly supported of size $N$. Taking the inverse Fourier transform of (60), we obtain the B-spline representation of the impulse response of the analysis filter

$$
\tilde{\varphi}(-t)=\sum_{k \in \mathbb{Z}} p[k] \tilde{\beta}(t-k)
$$

where $p[k]$ is the causal sequence whose $z$-transform is given by (59). The key observation now is that the infinite sum in the denominator of (20) represents the discrete-time Fourier transform of the sampled version of $\sum_{k \in \mathbb{Z}} p[k] \varphi_{1}(t-k)$, where $\varphi_{1}(t)=\tilde{\beta}(t) * \varphi(t)$. This yields an explicit formula for the $z$-transform of the inverse filter

$$
H_{\mathrm{LS}}(z)=\frac{1}{P(z) A_{1}(z)}
$$

where

$$
A_{1}(z)=\sum_{n \in \mathbb{Z}} \varphi_{1}(n) z^{-n} .
$$

Thus, to get the rational form of (63), all we need to do is evaluate the function $\varphi_{1}(t)$ at the integers, which can be done easily when $\varphi(t)$ is a B-spline, using the E-spline formalism presented in [35], [36].

The same technique also works for determining the transfer functions of the remaining filters. To this end, we introduce an additional B-spline function, $\beta_{\mathrm{L}}(t)=\beta_{\left(\lambda_{1}, \cdots, \lambda_{K}\right)}(t)$, whose Fourier domain expression is

$$
B_{\mathrm{L}}(f)=\prod_{k=1}^{K} \frac{1-e^{\lambda_{k}-j 2 \pi f}}{j 2 \pi f-\lambda_{k}}=\frac{\Delta_{\mathrm{L}}\left(e^{j 2 \pi f}\right)}{L(f)}
$$

where

$$
\Delta_{\mathrm{L}}(z)=\prod_{k=1}^{K}\left(1-e^{\lambda_{k}} z^{-1}\right) .
$$

We also define a few more auxiliary functions

$$
\begin{aligned}
& \varphi_{2}(t)=\varphi(-t) * \varphi(t) \\
& \varphi_{3}(t)=\tilde{\beta}(t) * \varphi(t) * \beta_{\mathrm{L}}(-t) * \beta_{\mathrm{L}}(t) \\
& \varphi_{4}(t)=\tilde{\beta}(-t) * \tilde{\beta}(t) * \beta_{\mathrm{L}}(-t) * \beta_{\mathrm{L}}(t) \\
& \varphi_{5}(t)=\mathrm{L}\{\varphi\}(-t) * \mathrm{~L}\{\varphi\}(t)=\mathrm{L}^{*} \mathrm{~L}\left\{\varphi_{2}\right\}(t) .
\end{aligned}
$$

Now, when $\varphi(t)$ is a B-spline, these functions are all exponential $\mathrm{B}$-splines, and they can be readily specified using the B-spline convolution relations given in [35]. As in the previous case, the only information that is really needed to determine the transfer functions of the various filters are the sample values of these functions at the integers. Specifically, we rewrite (27) and (36) as

$$
\begin{aligned}
& H_{\mathrm{TIK}}(z) \\
& =\frac{A_{1}\left(z^{-1}\right)}{P(z) A_{1}\left(z^{-1}\right) A_{1}(z)+\lambda \frac{S_{w}(z)}{P\left(z^{-1}\right)} \sum_{n \in \mathbb{Z}} \varphi_{5}(n) z^{-n}} \\
& H_{\mathrm{MX}}(z) \\
& =\frac{\sum_{n \in \mathbb{Z}} \varphi_{3}(n) z^{n}}{A_{\varphi}(z)\left(P(z) \sum_{n \in \mathbb{Z}} \varphi_{4}(n) z^{-n}+\frac{S_{w}(z)}{\sigma_{0}^{2} P\left(z^{-1}\right)} \Delta_{\mathrm{L}}(z) \Delta_{\mathrm{L}}\left(z^{-1}\right)\right)}
\end{aligned}
$$

where $A_{1}(z), P(z)$ are given by (64) and (59) respectively, and $A_{\varphi}(z)=\sum_{n \in \mathbb{Z}} \varphi_{2}(n) z^{-n}$, which is equivalent to (9). The key step for obtaining (67) is to multiply the numerator and denominator of (36) by the 1-periodic function $\left|\Delta_{\mathrm{L}}\left(e^{j 2 \pi f}\right)\right|^{2}$, which allows us to identify the Fourier transform of $\beta_{\mathrm{L}}(t)$ given by (65).

The various filter formulas call for the following comments. First, all sums over $n$ are finite because the auxiliary functions (exponential B-splines) are compactly supported. This means that the restoration filters $H_{\mathrm{LS}}(z), H_{\mathrm{TIK}}(z)$, and $H_{\mathrm{MX}}(z)$ are all rational. Second, in contrast with (27) and (36), the Tikhonov and minimax formulas (67) and (68) with $z=e^{j 2 \pi f}$ are completely safe numerically. The key point is that the numerator is bounded because the corresponding exponential $\mathrm{B}$-splines are well defined and compactly supported. This is not necessarily so for (36) because of the division by $L(f)$ which can be arbitrarily small (the trick here is that, by introducing the B-splines, we have been able to cancel all potentially dangerous poles and have moved them into the denominator). We also note that the denominator of (67) or (68) cannot vanish on the unit circle because it can always be written as $P(z) U(z)+V(z) / P\left(z^{-1}\right)$ where $U(z)$ and $V(z)$ are both positive definite (in particular, if $P\left(e^{j 2 \pi f}\right)$ gets very small, then the effect will be compensated by the second term becoming very large and vice versa). The least-squares filter (63), on the other hand, can become unstable if $A_{1}(z)$ has a zero close to the unit circle.

\section{B. Linking the Wiener and Minimax Solutions}

As mentioned in Section III-D, there is an equivalence between the minimax and Wiener solutions provided that we select $\mathrm{L}$ to be the whitening filter of the process $x(t)$. Restating this condition in terms of the quantities defined above, we have that

$$
S_{x}(f)=\frac{\sigma_{0}^{2}\left|B_{\mathrm{L}}(f)\right|^{2}}{\left|\Delta_{\mathrm{L}}\left(e^{j 2 \pi f}\right)\right|^{2}}=\frac{\sigma_{0}^{2}}{\prod_{k=1}^{K}\left|j 2 \pi f-\lambda_{k}\right|^{2}}
$$

where $B_{\mathrm{L}}(f)$ and $\Delta_{\mathrm{L}}\left(e^{j 2 \pi f}\right)$ are given by (65) and (66), respectively.

To be more concrete, let us consider the case of a first-order Markov process whose spectral power density is $S_{x}(f)=\sigma_{0}^{2} /\left((2 \pi f)^{2}+\log ^{2} \rho\right)$, where $0<\rho<1$ is the normalized correlation coefficient [37]. It is not difficult to see that the corresponding whitening operator is $\mathrm{L}=\mathrm{D}-\lambda_{1} \mathrm{I}$, with $\lambda_{1}=\log \rho<0$. This means that a first-order differential regularization operator is the best choice for this type of process and that the regularization parameters can be optimally matched to the spectral characteristics of the input signal; in this particular case $\lambda=1 / \sigma_{0}^{2}$, and $\lambda_{1}=\log \rho$. The argument obviously also holds for higher order Markov processes, which are "whitened" by higher order differential operators whose zeros are given by the "stable" poles of the spectral power density function; i.e., those that are in the left complex plane.

\section{Example: Sampling of a First-Order Differential System}

For illustration purposes, we consider the case of an analysis function that corresponds to a first-order differential system with a single pole at $\alpha_{1}=-1$. We are seeking a reconstruction in two spaces: i) the space of linear splines with $\varphi(t)=$ 


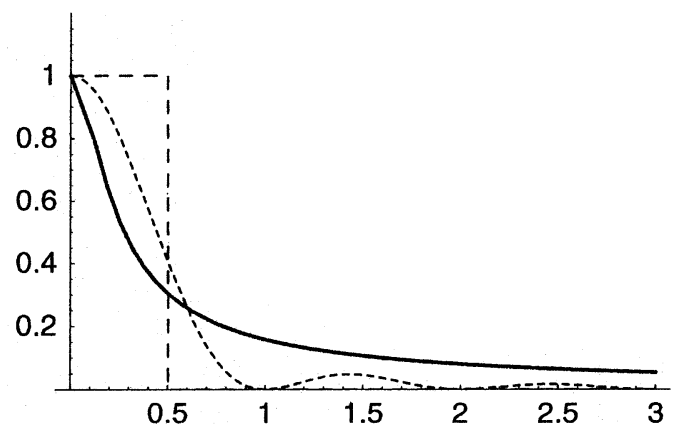

Fig. 5. Fourier transforms of the analysis function (solid line) and the two synthesis functions: linear spline (fine-dashed line) and bandlimited (dashed line).

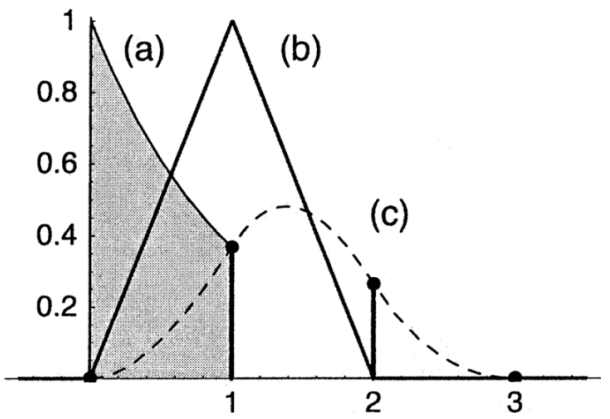

Fig. 6. Basis functions associated with a linear spline signal reconstruction: (a) the analysis B-spline $\tilde{\beta}(t)$ (first-order exponential spline); (b) the synthesis function $\varphi(t)$ (solid line); and (c) the auxiliary function $\varphi_{1}(t)=\tilde{\beta}(t) * \varphi(t)$ (dashed line). The key point is that these are all compactly supported. The integer samples of $\varphi_{1}(t)$ are the coefficients of the Laurent polynomial $A_{1}(z)$ that appears in the specification of the least-squares and Tikhonov reconstruction filters.

$\beta_{(0,0)}(t)$ (causal triangle) and ii) the space of bandlimited functions with $\varphi(t)=\operatorname{sinc}(t)$. The Fourier transforms of these various functions are shown in Fig. 5. The function $L(f)$ is chosen as the first-order differential regularization operator $\mathrm{L}=$ $\mathrm{D}-\lambda_{1} \mathrm{I}$, which is "optimal" for a first-order Markov process. We assume that the measurements are corrupted by standardized white noise, corresponding to the choice $S_{w}\left(e^{j 2 \pi f}\right)=1$.

Some of the relevant basis functions for the linear spline case are shown in Fig. 6. The impulse response of the analysis filter is a causal exponential that can be represented using the first-order exponential B-spline, $\tilde{\beta}(t)=\beta_{(-1)}(t)$, shown in Fig. 6(a). The corresponding sequence of weights in (62) is the discrete version of this filter, $p[k]=e^{-k}$, with $k \geq 0$, whose $z$-transform is given by (59) with $N=1$ and $\alpha_{1}=-1$. The auxiliary functions, $\left\{\varphi_{i}(t)\right\}_{i=1, \cdots, 5}$, are all exponential B-splines that can be determined analytically [35]. For instance, $\varphi_{2}(t)=$ $\beta_{(-1)}(t) * \beta_{(0,0)}(t)=\beta_{(-1,0,0)}(t)$. Sampling this function at the integers yields $A_{1}(z)=0.367879 z^{-1}+0.264241 z^{-2}$, as illustrated in Fig. 6(c). By substituting these expressions in (63), we get the following concise, rational form of the least-squares filter

$$
H_{\mathrm{LS}}(z)=z \frac{1-e^{-1} z^{-1}}{0.367879+0.264241 z^{-1}}
$$

which is causal and stable and can be implemented recursively. The same technique is also applicable to the determination of the rational forms of the corresponding Tikhonov and minimax (or

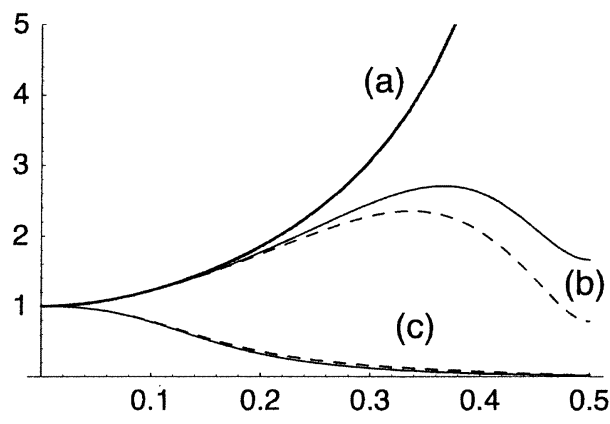

Fig. 7. Frequency responses (amplitude) of various filters associated with a linear spline signal reconstruction: (a) least-squares (inverse) filter; (b) Tikhonov (solid line) and minimax (dashed line) filters for $\lambda=1 / \sigma_{0}^{2}=10^{-2}$; and (c) Tikhonov and minimax filters for $\lambda=1 / \sigma_{0}^{2}=1$. Solutions (b) and (c) are regularized by means of the same first-order operator L. The minimax filter is identical to the Wiener filter that would be associated with a first-order Markov process whose spectral power density is given by (69).

Wiener) filters. The frequency responses of these filters for different values of $\lambda$ and $\lambda_{1}=10^{-6}$ are shown in Fig. 7. We note that the least-squares solution performs the strongest enhancement of the higher frequency part of the spectrum. The Tikhonov and minimax filters that we are displaying have matched parameters - same regularization operator $\mathrm{L}$ and $\lambda=1 / \sigma_{0}^{2}$. The minimax filter results in a slightly stronger attenuation of the higher frequencies. By increasing $\lambda$, one can modify the frequency responses of the two filters and make them more and more low pass. Conversely, when $\lambda$ tends to zero, the Tikhonov filter converges to the least-squares solution.

In a practical situation, the regularization parameter $\lambda$ should be matched to the noise level. In fact, the functional equivalence between the various solutions suggests that $\lambda$ should be chosen to be inversely proportional to the signal-to-noise ratio, which is itself proportional to $\sigma_{0}^{2} / \sigma^{2}$, where $\sigma^{2}$ is the noise variance.

The frequency responses of the corresponding filters for a bandlimited reconstruction (cf. (46), (47) and (50)) are shown in Fig. 8 for comparison. The trend is essentially the same as in the linear spline case. The inverse filtering effect, however, is not quite as strong as in Fig. 7. This is not surprising since the bandlimited solution is compensating for the effect of the input filter (e.g., $\widetilde{\Phi}^{*}(f)$ ) alone, while the spline solution also takes into account the nonideal frequency response of the synthesis function (cf. Fig. 5).

We observe that, in both cases, the Tikhonov and minimax filters have the same qualitative behavior, but they are not identical. This is to be expected because the underlying cost functions are quite distinct. However, comparing (67) and (68), it can be seen that the formulas are not so fundamentally different from each other. In fact, the two solutions can be made equivalent by choosing the particular synthesis function

$$
\varphi(t)=\tilde{\beta}(-t) * \beta_{\mathrm{L}}(t) * \beta_{\mathrm{L}}(-t) .
$$

The link between both approaches can also be made explicit in the bandlimited case, as discussed in Section IV-A. Indeed, the Tikhonov filter (47) (cf. continuous line in Fig. 8) is equivalent to a hypothetical minimax solution associated with a bandlimited analysis filter: $\operatorname{rect}(f) \cdot \widetilde{\Phi}^{*}(f)$ (cf. (50)). Alternatively, it also corresponds to the Wiener solution of a modified version of the initial problem where the input signal is bandlimited, that 


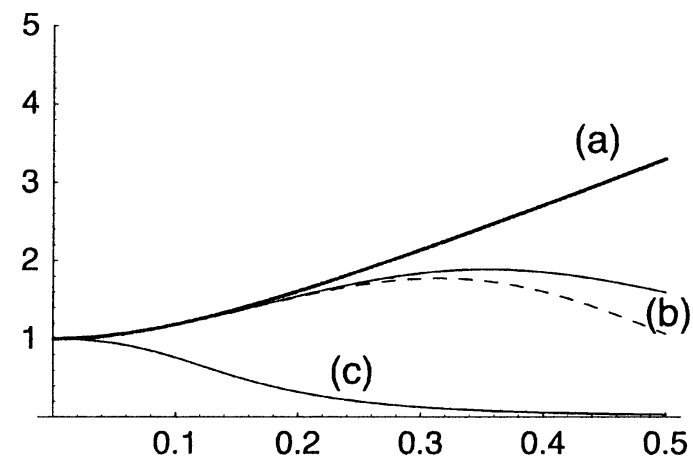

Fig. 8. Frequency responses (amplitude) of various filters associated with a bandlimited signal reconstruction: (a) least-squares (inverse) filter; (b) Tikhonov (solid line) and minimax (dashed line) filters for a medium SNR ratio regime; and (c) Tikhonov and minimax filters (undistinguishable) for a lower SNR regime. The regularization parameters for (b) and (c) are the same as in Fig. 7. The minimax filter (dashed line) is identical to the Wiener filter that would be associated with a first-order Markov process whose spectral power density is given by (69). Likewise, the Tikhonov filter (solid line) corresponds to the Wiener solution associated with a bandlimited version of the first-order Markov process.

is, when $S_{x}(f)$ is replaced by rect $(f) \cdot S_{x}(f)$. Thus, it can make sense to use the Tikhonov filter as a practical substitute for the minimax or Wiener solutions, especially since it is generally simpler to specify numerically: The function $\varphi_{5}(t)$ depends directly on $\varphi(t)$, while the evaluation of $\varphi_{3}(t)$ and $\varphi_{4}(t)$ involve more complex interactions between the analysis, regularization and synthesis basis functions.

\section{CONCLUSION}

In this paper, we considered the recovery of a continuoustime signal $x(t)$ given noisy samples of its filtered version. The signal reconstruction is performed in a "shift-invariant" space and is specified by a sequence of weights $c[n]$ in a generic basis $\{\varphi(t-n)\}_{n \in \mathbb{Z}}$ that is defined in the continuous-time domain. Our formulation addresses the noisy data interpolation and the deconvolution problems simultaneously.

The solutions that we have identified all use linear digital filtering and were derived based on the minimization of a suitable cost function. The proposed methods differ in the assumptions that have been made and are summarized in Table I. The simplest design criteria is minimizing the least-squares error which results in an inverse filter. It corresponds to the oblique projection method described in [8] for consistent sampling. The main feature of this solution is that the estimate remains unchanged if it is reinjected in the noise-free system, meaning that the data is fitted exactly. The down side of this strategy is that is provides a poor signal estimate for noisy data because of its tendency to overamplify measurement noise.

The second approach is Tikhonov regularization, which introduces a smoothness constraint on the signal to counterbalance the effect of noise. The criterion to be minimized is a data term + regularizations. Its free parameters are the regularization operator $\mathrm{L}$ and the regularization factor $\lambda$, which should be set inversely proportional to the signal-to-noise ratio. The least-squares solution is recovered when $\lambda \rightarrow 0$.

The third approach is the minimax filter, which assumes that the signal is deterministic and corrupted by stochastic noise. It uses the a priori knowledge that $\|\mathrm{L} x\|_{L_{2}} \leq \sigma_{0}$, where $\mathrm{L}$ is a suitable operator. The solution is optimal in the sense that it minimizes the worst-case projected MSE over the set of admissible signals. We developed two versions of the minimax problem (projected pointwise and average) that yield equivalent filters.

The final approach is the adaptation of the Wiener filter to our particular context where the signal is continuously defined and the noise discrete. Here, the assumption is that both are realizations of stationary processes with known spectral power densities, and the criterion that is minimized is the projected MSE.

In the high SNR regime, the minimax and Wiener filters result in the reconstructed signal $\hat{x}(t)=P_{\mathcal{V}} P_{\widetilde{\mathcal{V}}} x(t)$, while the leastsquares and Tikhonov filters lead to $\hat{x}(t)=E_{\mathcal{V}_{\mathcal{V}}{ }^{\perp}} x(t)$.

The choice of the most suitable method clearly depends on the context (e.g., deterministic versus stochastic) and on the type of a priori information available. Nevertheless, by comparing the various solutions, we have been able to make some interesting links and have come up with simple guidelines for selecting the free parameters of the various algorithms. Obviously, the least-squares filter is only adequate when the measurement noise is negligible. Otherwise, one would tend to favor the minimax or Wiener solutions, depending on whether the signal should be viewed as deterministic or stochastic, because both minimize a MSE criterion that is a direct measure of the quality of the solution. The mathematical equivalence between the minimax and Wiener formulas suggests selecting an "optimal" operator L that "whitens" the signal. In practice, this operator may be determined from the signal using some form of spectral estimation. Even though the use of the Tikhonov formulation is somewhat ad hoc and more difficult to justify on statistical grounds, it is not too different from the minimax and Wiener methods, provided that one uses the appropriate regularization. In practice, it should perform quite adequately if we select the same "whitening" operator L as for the minimax solution, choose a spectral weighting function that is inversely proportional to the power spectrum of the noise, and if we set $\lambda=1 / \sigma_{0}^{2}$ with $\sigma_{0}^{2} \approx\|\mathrm{L} x\|_{L_{2}}$ where $x$ is the noise-free signal. In fact, we have shown that the last three (non least-squares) filters can be made rigorously equivalent, if the various parameters and the reconstruction space $V(\varphi)$ are chosen appropriately.

\section{APPENDIX I \\ DERIVATION OF THE TIKHONOV FILTER}

To develop the Tikhonov filter, we first note that using (13) the set $\mathcal{G}$ can be expressed as

$$
\mathcal{G}=\left\{x(t): \int_{0}^{1} \sum_{k \in \mathbb{Z}}|L(f+k)|^{2}|X(f+k)|^{2} d f \leq \sigma_{0}^{2}\right\}
$$

which will be convenient in our derivations below.

Differentiating $\epsilon_{\mathrm{TIK}}$ with respect to $H\left(e^{j 2 \pi f}\right)$ and equating to 0

$$
\begin{aligned}
S_{w}^{-1}\left(e^{j 2 \pi f}\right) & \left(1-H\left(e^{j 2 \pi f}\right) A_{\tilde{\varphi} \varphi}\left(e^{j 2 \pi f}\right)\right) A_{\tilde{\varphi} \varphi}\left(e^{j 2 \pi f}\right) \\
& =\lambda H\left(e^{j 2 \pi f}\right) \sum_{k \in \mathbb{Z}}|L(f+k)|^{2}|\Phi(f+k)|^{2}
\end{aligned}
$$


for all $f$ such that $Y\left(e^{j 2 \pi f}\right) \neq 0$, where $A_{\tilde{\varphi} \varphi}\left(e^{j 2 \pi f}\right)$ is defined by (19). If $\lambda=0$, then to satisfy (72) we must have

$$
H\left(e^{j 2 \pi f}\right)=\frac{1}{A_{\tilde{\varphi} \varphi}\left(e^{j 2 \pi f}\right)}, \quad f \in \Omega
$$

where $\Omega$ denotes the set of frequencies $f \in[-1 / 2,1 / 2]$ such that $Y\left(e^{j 2 \pi f}\right) A_{\tilde{\varphi} \varphi}\left(e^{j 2 \pi f}\right) \neq 0$. Note that $H\left(e^{j 2 \pi f}\right)$ is arbitrary on $f \notin \Omega$. The solution (73) is valid only if the resulting $\hat{x}(t)$ satisfies the constraint $\hat{x}(t) \in \mathcal{G}$. For example, if we choose $H\left(e^{j 2 \pi f}\right)=0$ for $f \notin \Omega$, then the solution holds only if

$$
\int_{f \in \Omega} \sum_{k \in \mathbb{Z}}|L(f+k)|^{2}|\Phi(f+k)|^{2} \frac{|Y(f+k)|^{2}}{\left|A_{\tilde{\varphi} \varphi}\left(e^{j 2 \pi f}\right)\right|^{2}} d f \leq \sigma_{0}^{2} .
$$

If (74) is not satisfied, then $\lambda>0$, and the optimal $H\left(e^{j 2 \pi f}\right)$ is

$$
\begin{aligned}
& H_{\mathrm{TIK}}\left(e^{j 2 \pi f}\right) \\
& =\frac{A_{\tilde{\varphi} \varphi}\left(e^{j 2 \pi f}\right)}{\left|A_{\tilde{\varphi} \varphi}\left(e^{j 2 \pi f}\right)\right|^{2}+\lambda S_{w}\left(e^{j 2 \pi f}\right) \sum_{k \in \mathbb{Z}}|L(f+k)|^{2}|\Phi(f+k)|^{2}}
\end{aligned}
$$

for frequencies $f$ for which $Y\left(e^{j 2 \pi f}\right) \neq 0$, where $\lambda>0$ is chosen such that

$$
\begin{aligned}
\int_{0}^{1}\left|Y\left(e^{j 2 \pi f}\right)\right|^{2} \mid & \left.H_{\mathrm{TIK}}\left(e^{j 2 \pi f}\right)\right|^{2} \times \\
& \sum_{k \in \mathbb{Z}}|L(f+k)|^{2}|\Phi(f+k)|^{2} d f=\sigma_{0}^{2} .
\end{aligned}
$$

\section{APPENDIX II}

PROOF OF LEMMA 1

The projected MSE is given by

$$
\begin{aligned}
& \mathrm{E}\left\{\left|\hat{x}\left(t_{0}\right)-x_{\mathcal{V}}\left(t_{0}\right)\right|^{2}\right\}=\left|\sum_{n \in \mathbb{Z}}\left(h * y_{0}\right)[n] \varphi\left(t_{0}-n\right)-x_{\mathcal{V}}\left(t_{0}\right)\right|^{2} \\
&+\mathrm{E}\left\{\left|\sum_{n \in \mathbb{Z}}(h * w)[n] \varphi\left(t_{0}-n\right)\right|^{2}\right\}
\end{aligned}
$$

We first consider the expression $\mid \sum_{n \in \mathbb{Z}}\left(h * y_{0}\right)[n] \varphi\left(t_{0}-\right.$ $n)-\left.x_{\mathcal{V}}\left(t_{0}\right)\right|^{2}$. To this end we note that $B_{t_{0}}\left(e^{j 2 \pi f}\right)$ of (32) is the discrete-time Fourier transform of the sequence $b_{t_{0}}[n]=$ $\varphi\left(t_{0}+n\right)$. Using (11), we have that

$$
\begin{aligned}
\sum_{n \in \mathbb{Z}} & \left(h * y_{0}\right)[n] \varphi\left(t_{0}-n\right) \\
= & \left\langle\left(h * y_{0}\right)[n], b_{t_{0}}[-n]\right\rangle_{\ell_{2}} \\
= & \int_{0}^{1} H\left(e^{j 2 \pi f}\right) Y_{0}\left(e^{j 2 \pi f}\right) B_{t_{0}}\left(e^{j 2 \pi f}\right) d f \\
= & \int_{0}^{1} H\left(e^{j 2 \pi f}\right) B_{t_{0}}\left(e^{j 2 \pi f}\right) \\
& \times \sum_{\ell \in \mathbb{Z}} X(f+\ell) \widetilde{\Phi}^{*}(f+\ell) d f
\end{aligned}
$$

where the last equality follows from (7).
Denoting by $X_{\mathcal{V}}(f)$ the Fourier transform of $x_{\mathcal{V}}(t)$

$$
X_{\mathcal{V}}(f)=\frac{\sum_{k \in \mathbb{Z}} \Phi^{*}(f+k) X(f+k)}{A_{\varphi}\left(e^{j 2 \pi f}\right)} \Phi(f)
$$

where $A_{\varphi}\left(e^{j 2 \pi f}\right)$ is given by (9) [13]. Therefore

$$
\begin{aligned}
x_{\mathcal{V}}\left(t_{0}\right) & =\int_{-\infty}^{+\infty} X_{\mathcal{V}}(f) e^{j 2 \pi f t_{0}} d f \\
& =\int_{0}^{1} \sum_{k \in \mathbb{Z}} X_{\mathcal{V}}(f+k) e^{j 2 \pi(f+k) t_{0}} d f \\
& =\int_{0}^{1} B_{t_{0}}\left(e^{j 2 \pi f}\right) \frac{\sum_{k \in \mathbb{Z}} \Phi^{*}(f+k) X(f+k)}{A_{\varphi}\left(e^{j 2 \pi f}\right)} d f .
\end{aligned}
$$

Combining (78) and (80),

$$
\begin{aligned}
&\left|\sum_{n \in \mathbb{Z}}\left(h * y_{0}\right)[n] \varphi\left(t_{0}-n\right)-x_{\mathcal{V}}\left(t_{0}\right)\right|^{2} \\
& \quad=\left|\int_{0}^{1} B_{t_{0}}\left(e^{j 2 \pi f}\right) \sum_{k \in \mathbb{Z}} X(f+k) Z_{t_{0}}(f+k) d f\right|^{2} .
\end{aligned}
$$

Next, we note that

$$
\begin{aligned}
& E\left\{\left|\sum_{n \in \mathbb{Z}}(h * w)[n] \varphi\left(t_{0}-n\right)\right|^{2}\right\} \\
&=\mathrm{E}\left\{\left|\sum_{n \in \mathbb{Z}}(h * w)[n] b_{t_{0}}[-n]\right|^{2}\right\}
\end{aligned}
$$

and

$$
\begin{aligned}
& \mathrm{E}\left\{\left|\sum_{n \in \mathbb{Z}}(h * w)[n] b_{t_{0}}[-n]\right|^{2}\right\} \\
& =\mathrm{E}\left\{\left|\left(h * w * b_{t_{0}}\right)[0]\right|^{2}\right\} \\
& =\int_{0}^{1}\left|H\left(e^{j 2 \pi f}\right)\right|^{2}\left|B_{t_{0}}\left(e^{j 2 \pi f}\right)\right|^{2} S_{w}\left(e^{j 2 \pi f}\right) d f
\end{aligned}
$$

completing the proof of the proposition.

\section{APPENDIX III}

PROOF OF LEMMA 2

To prove the lemma we first note that

$$
\begin{aligned}
& \mid \begin{aligned}
\left|\int_{0}^{1} \sum_{k \in \mathbb{Z}} X(f+k) Z(f+k) d f\right| \\
\leq \int_{0}^{1} \sum_{k \in \mathbb{Z}}|X(f+k)||Z(f+k)| d f
\end{aligned}
\end{aligned}
$$

with equality if $\psi_{X}(f)=-\psi_{Z}(f)$, where $X(f)=$ $|X(f)| e^{j \psi_{X}(f)}$ and $Z(f)=|Z(f)| e^{j \psi_{Z}(f)}$ so that $\psi_{X}(f)$ and $\psi_{Z}(f)$ are the phases of $X(f)$ and $Z(f)$ respectively. Since the constraint set $\mathcal{G}$ does not depend on the phase of $X(f)$, it 
follows from (84) that the phase of the maximizing $X(f)$ is equal to $\psi_{X}(f)=-\psi_{Z}(f)$, and $X(f) Z(f)=|X(f) Z(f)|$. Thus a

$$
\begin{aligned}
\max _{x(t) \in \mathcal{G}}\left|\int_{0}^{1} \sum_{k \in \mathbb{Z}} X(f+k) Z(f+k) d f\right|^{2} \\
=\max _{x(t) \in \mathcal{G}}\left|\int_{0}^{1} \sum_{k \in \mathbb{Z}}\right| X(f+k)|| Z(f+k)|d f|^{2} .
\end{aligned}
$$

Now

$$
\begin{aligned}
\left|\int_{0}^{1} \sum_{k \in \mathbb{Z}}\right| X(f+k)|| Z(f+k)|d f|^{2} \\
\quad=\left|\int_{-\infty}^{+\infty}\right| L(f)|| X(f)|| Z(f)|/| L(f)|d f|^{2} \\
\leq \int_{-\infty}^{+\infty}|L(f)|^{2}|X(f)|^{2} d f \int_{-\infty}^{+\infty}|Z(f)|^{2} / \mid L(f)^{2} d f \\
\leq \sigma_{0}^{2} \int_{0}^{1} \sum_{k \in \mathbb{Z}}|Z(f+k)|^{2} /|L(f+k)|^{2} d f
\end{aligned}
$$

where the first inequality follows from applying Cauchy-Schwarz, and the second inequality holds for any $x(t) \in \mathcal{G}$. We have equality in (86) if

$$
|X(f)|=\frac{\sigma_{0}|Z(f)| /|L(f)|^{2}}{\left(\int_{0}^{1} \sum_{k \in \mathbb{Z}}|Z(f+k)|^{2} /|L(f+k)|^{2} d f\right)^{\frac{1}{2}}}
$$

which satisfies the constraint $\int_{-\infty}^{+\infty}|L(f)|^{2}|X(f)|^{2} d f \leq \sigma_{0}^{2}$, completing the proof.

\section{APPENDIX IV}

\section{DERIVATION OF THE AVERAGE Minimax MSE FILTER}

To develop the solution to (43), we first compute the integral $\int_{0}^{1} M\left(t_{0}\right) d t_{0}$. To this end, we need to evaluate the following two integrals:

$$
\begin{aligned}
I_{1}(f) & =\int_{0}^{1}\left|B_{\tau}\left(e^{j 2 \pi f}\right)\right|^{2} d \tau \\
& =\int_{0}^{1}\left|\sum_{\ell \in \mathbb{Z}} \Phi(f+\ell) e^{j 2 \pi \ell \tau}\right|^{2} d \tau \\
I_{2}(f, k)= & \int_{0}^{1}\left|Z_{\tau}(f+k)\right|^{2} d \tau \\
= & \int_{0}^{1} \mid \widetilde{\Phi}^{*}(f+k) H\left(e^{j 2 \pi f}\right) \\
& \times \sum_{\ell \in \mathbb{Z}} \Phi(f+\ell) e^{j 2 \pi \ell \tau}-\left.e^{j 2 \pi k \tau}\right|^{2} d \tau .
\end{aligned}
$$

Using the identity

$$
\int_{0}^{1} e^{j 2 \pi(\ell-k) \tau} d \tau= \begin{cases}1, & k=\ell \\ 0, & k \neq \ell\end{cases}
$$

we have that

$I_{1}(f)=\sum_{k, \ell \in \mathbb{Z}} \Phi(f+\ell) \Phi^{*}(f+k) \int_{0}^{1} e^{j 2 \pi(\ell-k) \tau} d \tau=A_{\varphi}\left(e^{j 2 \pi f}\right)$

and

$$
\begin{aligned}
I_{2}(f, k)=\mid \widetilde{\Phi}^{*}( & f+k)\left.H\left(e^{j 2 \pi f}\right)\right|^{2} A_{\varphi}\left(e^{j 2 \pi f}\right)+1 \\
& -2 \Re\left\{\widetilde{\Phi}^{*}(f+k) \Phi(f+k) H\left(e^{j 2 \pi f}\right)\right\} .
\end{aligned}
$$

From (88), (90), and (91)

$$
\begin{aligned}
\int_{0}^{1} M\left(t_{0}\right) d t_{0}= & \int_{0}^{1}\left(\left|H\left(e^{j 2 \pi f}\right)\right|^{2} S_{w}\left(e^{j 2 \pi f}\right) A_{\varphi}\left(e^{j 2 \pi f}\right)\right. \\
& \left.+\sigma_{0}^{2} \sum_{k \in \mathbb{Z}} I_{2}(f, k) /|L(f+k)|^{2}\right) d f
\end{aligned}
$$

where we assume that $\sum_{k \in \mathbb{Z}} 1 /|L(f+k)|^{2}<+\infty$.

Since the integrand in (92) is nonnegative, the optimal solution can be found by minimizing the integrand, which is convex in $H\left(e^{j 2 \pi f}\right)$. Differentiating (92) with respect to $H\left(e^{j 2 \pi f}\right)$ and equating to zero, the optimal filter that is the solution to (43) is given by (36).

\section{APPENDIX V}

PROOF OF THEOREM 2

In the stochastic setting, the MSE is given by

$$
\begin{aligned}
\mathrm{E}\left\{\left|\hat{x}\left(t_{0}\right)-x_{\mathcal{V}}\left(t_{0}\right)\right|^{2}\right\} \\
=\mathrm{E}\left\{\left|\sum_{n \in \mathbb{Z}}\left(h * y_{0}\right)[n] \varphi\left(t_{0}-n\right)-x_{\mathcal{V}}\left(t_{0}\right)\right|^{2}\right\} \\
+\mathrm{E}\left\{\left|\sum_{n \in \mathbb{Z}}(h * w)[n] \varphi\left(t_{0}-n\right)\right|^{2}\right\} .
\end{aligned}
$$

To compute the left-hand expression in (93), we first express $x_{\mathcal{V}}\left(t_{0}\right)$ in the time domain. Taking the inverse Fourier transform of (79)

$$
x_{\mathcal{V}}\left(t_{0}\right)=\sum_{n \in \mathbb{Z}}(d * a)[n] \varphi\left(t_{0}-n\right)
$$


where $d[n]=\langle\varphi(t-n), x(t)\rangle$ and $a[n]$ is the inverse Fourier transform of $1 / A_{\varphi}\left(e^{j 2 \pi f}\right)$. Therefore

$$
\begin{aligned}
\mathrm{E}\left\{\left|\sum_{n \in \mathbb{Z}}\left(h * y_{0}\right)[n] \varphi\left(t_{0}-n\right)-x_{\mathcal{V}}\left(t_{0}\right)\right|^{2}\right\} \\
=\mathrm{E}\left\{\left|\sum_{n \in \mathbb{Z}}\left(h * y_{0}-d * a\right)[n] \varphi\left(t_{0}-n\right)\right|^{2}\right\} \\
=\mathrm{E}\left\{\left|\left(\left(h * y_{0}-d * a\right) * b_{t_{0}}\right)[0]\right|^{2}\right\} \\
=\int_{0}^{1}\left|B_{t_{0}}\left(e^{j 2 \pi f}\right)\right|^{2} \sum_{k \in \mathbb{Z}} S_{x}(f+k) \\
\quad \times\left|\widetilde{\Phi}^{*}(f+k) H\left(e^{j 2 \pi f}\right)-\frac{\Phi^{*}(f+k)}{A_{\varphi}\left(e^{j 2 \pi f}\right)}\right|^{2} d f
\end{aligned}
$$

where $b_{t_{0}}[n]=\varphi\left(t_{0}+n\right)$ is the inverse Fourier transform of $B_{t_{0}}\left(e^{j 2 \pi f}\right)$ given by (32). Combining (95) with (83) (in Appendix II)

$$
\begin{aligned}
& \mathrm{E}\left\{\left|\hat{x}\left(t_{0}\right)-x_{\mathcal{V}}\left(t_{0}\right)\right|^{2}\right\}=\int_{0}^{1} d f\left|B_{t_{0}}\left(e^{j 2 \pi f}\right)\right|^{2} \\
& \cdot\left(\sum_{k \in \mathbb{Z}} S_{x}(f+k)\left|\widetilde{\Phi}^{*}(f+k) H\left(e^{j 2 \pi f}\right)-\frac{\Phi^{*}(f+k)}{A_{\varphi}\left(e^{j 2 \pi f}\right)}\right|^{2}\right. \\
& \left.\quad+\left|H\left(e^{j 2 \pi f}\right)\right|^{2} S_{w}\left(e^{j 2 \pi f}\right)\right) .
\end{aligned}
$$

Therefore, the Wiener filter is the solution to

$$
\begin{aligned}
& \min _{H\left(e^{j 2 \pi f}\right)}\left\{\sum_{k \in \mathbb{Z}} S_{x}(f+k)\left|\widetilde{\Phi}^{*}(f+k) H\left(e^{j 2 \pi f}\right)-\frac{\Phi^{*}(f+k)}{A_{\varphi}\left(e^{j 2 \pi f}\right)}\right|^{2}\right. \\
& \left.+\left|H\left(e^{j 2 \pi f}\right)\right|^{2} S_{w}\left(e^{j 2 \pi f}\right)\right\} .
\end{aligned}
$$

Since the objective of (97) is convex in $H\left(e^{j 2 \pi f}\right)$, the optimal filter can be found by setting the derivative to 0 , which results in (44).

\section{REFERENCES}

[1] N. Wiener, The Extrapolation, Interpolation and Smoothing of Stationary Time Series. New York: Wiley, 1949.

[2] A. Kolmogorov, "Interpolation and extrapolation," Bull. Acad. Sci. U.S.S.R., Math. Ser., vol. 5, pp. 3-14, 1941.

[3] C. E. Shannon, "Communication in the presence of noise," in Proc. I.R.E., vol. 37, 1949, pp. 10-21.

[4] A. J. Jerri, "The Shannon sampling theorem-its various extensions and applications: A tutorial review," Proc. IEEE, vol. 65, no. 11, pp. 1565-1596, 1977.

[5] M. Unser, "Sampling—50 years after Shannon," Proc. IEEE, vol. 88, no. 4, pp. 569-587, 2000.

[6] E. Meijering, "A chronology of interpolation: from ancient astronomy to modern signal and image processing," Proc. IEEE, vol. 90, no. 3, pp. 319-342, 2002

[7] M. Unser and T. Blu, "Generalized smoothing splines and the optimal discretization of the Wiener filter," IEEE Trans. Signal Process., vol. 53, no. 6, pp. 2146-2159, Jun. 2006.

[8] M. Unser and A. Aldroubi, "A general sampling theory for nonideal acquisition devices," IEEE Trans. Signal Process., vol. 42, no. 11, pp. 2915-2925, Nov. 1994.
[9] Y. C. Eldar, "Sampling and reconstruction in arbitrary spaces and oblique dual frame vectors," J. Fourier Anal. Appl., vol. 1, no. 9, pp. 77-96, Jan. 2003.

[10] _ - "Sampling without input constraints: Consistent reconstruction in arbitrary spaces," in Sampling, Wavelets and Tomography, A. I. Zayed and J. J. Benedetto, Eds. Boston, MA: Birkhauser, 2004, pp. 33-60.

[11] Y. C. Eldar and T. Werther, "General framework for consistent sampling in Hilbert spaces," Int. J. Wavelets, Multiresolution Inf. Process., vol. 3 , no. 3, pp. 347-359, Sep. 2005.

[12] Y. C. Eldar and T. Dvorkind, "A minimum squared-error framework for sampling and reconstruction in arbitrary spaces," IEEE Trans. Signal Process., vol. 54, no. 6, pp. 2155-2167, Jun. 2006.

[13] A. Aldroubi and M. Unser, "Sampling procedures in function spaces and asymptotic equivalence with Shannon's sampling theory," Numer. Funct. Anal. Optim., vol. 15, no. 1-2, pp. 1-21, Feb. 1994.

[14] A. Aldroubi and K. Gröchenig, "Nonuniform sampling and reconstruction in shift invariant spaces," SIAM Rev., vol. 43, pp. 585-620, 2001.

[15] K. F. Cheung and R. J. Marks II, "Ill-posed sampling theorems," IEEE Trans. Circuits Syst., vol. CAS-32, pp. 481-484, May 1985.

[16] D. S. Chen and J. P. Allebach, "Analysis of error in reconstruction of two-dimensional signals from irregularly spaced samples," IEEE Trans. Acoust., Speech, Signal Process., vol. ASSP-35, no. 2, pp. 173-180, Feb. 1987.

[17] G. Wan and W. Han, "Minimum error bound of signal reconstruction," IEEE Signal Process. Lett., vol. 6, no. 12, pp. 309-311, Dec. 1999.

[18] H. Johansson and P. Lowenborg, "Reconstruction of nonuniformly sampled bandlimited signals by means of digital fractional delay filters," IEEE Trans. Signal Process., vol. 50, no. 11, pp. 2757-2767, Nov. 2002.

[19] E. J. Diethorn and D. C. Munson Jr., "A linear, time-varying system framework for noniterative discrete-time band-limited signal extrapolation," IEEE Trans. Signal Process., vol. 39, no. 1, pp. 55-68, Jan. 1991.

[20] A. Krzyzak, E. Rafajlowicz, and M. Pawlak, "Moving average restoration of bandlimited signals from noisy observations," IEEE Trans. Signal Processing, vol. 45, no. 12, pp. 2967-2976, Dec. 1997.

[21] M. Pawlak, E. Rafajlowicz, and A. Krzyzak, "Postfiltering versus prefiltering for signal recovery from noisy samples," IEEE Trans. Inf. Theory, vol. 49, no. 12, pp. 3195-3212, Dec. 2003.

[22] G. Demoment, "Image reconstruction and restoration-Overview of common estimation structures and problems," IEEE Trans. Acoust., Speech, Signal Process., vol. 37, no. 12, pp. 2024-2036, Dec. 1989.

[23] N. B. Karayiannis and A. N. Venetsanopoulos, "Regularization theory in image restoration-The stabilizing functional approach," IEEE Trans. Acoust., Speech, Signal Process., vol. 38, no. 7, pp. 1155-1179, Jul. 1990.

[24] M. R. Banham and A. K. Katsaggelos, "Digital image restoration," IEEE Signal Process. Mag., vol. 14, no. 2, pp. 24-41, Mar. 1997.

[25] Y. C. Eldar, A. Ben-Tal, and A. Nemirovski, "Linear minimax regret estimation of deterministic parameters with bounded data uncertainties," IEEE Trans. Signal Process., vol. 52, no. 8, pp. 2177-2188, Aug. 2004.

[26] — - "Robust mean-squared error estimation in the presence of model uncertainties," IEEE Trans. Signal Process., vol. 53, no. 1, pp. 168-181, Jan. 2005.

[27] O. Christensen and Y. C. Eldar, "Oblique dual frames and shift-invariant spaces," Appl. Comput. Harmon. Anal., vol. 17, no. 1, pp. 48-68, Jul. 2004.

[28] T. Blu and M. Unser, "Approximation error for quasi-interpolators and (multi-) wavelet expansions," Appl. Comput. Harmon. Anal., vol. 6, no. 2, pp. 219-251, Mar. 1999.

[29] J. J. Benedetto, Harmonic Analysis and Applications. Boca Raton, FL: CRC, 1997.

[30] A. K. Louis, "A unified approach to regularization methods for linear ill-posed problems," Inverse Probl., vol. 15, pp. 489-498, 1999.

[31] D. P. Bertsekas, Nonlinear Programming, 2nd ed. Belmont, MA: Athena Scientific, 1999

[32] Y. C. Eldar, "Robust deconvolution of deterministic and random signals," IEEE Trans. Inf. Theory, vol. 51, no. 8, pp. 2921-2929, Aug. 2005.

[33] I. J. Schoenberg, "Spline functions and the problem of graduation," Proc. Nat. Acad. Sci., vol. 52, pp. 947-950, 1964.

[34] T. Poggio, V. Torre, and C. Koch, "Computational vision and regularization theory," Nature, vol. 317, pp. 314-319, 1985.

[35] M. Unser, "Cardinal exponential splines: Part II-Think analog, act digital," IEEE Trans. Signal Process., vol. 53, no. 4, pp. 1439-1449, Apr. 2005.

[36] M. Unser and T. Blu, "Cardinal exponential splines: Part I-Theory and filtering algorithms," IEEE Trans. Signal Process., vol. 53, no. 4, pp. 1425-1438, Apr. 2005.

[37] A. Papoulis, Probability, Random Variables, and Stochastic Processes, 3rd ed. New York: McGraw-Hill, 1991. 


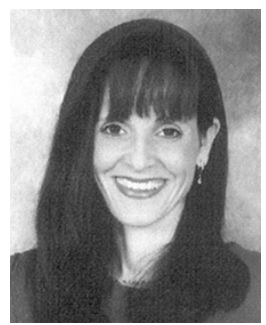

Yonina C. Eldar (S'98-M'02) received the B.Sc. degree in physics in 1995 and the B.Sc. degree in electrical engineering in 1996, both from Tel-Aviv University (TAU), Tel-Aviv, Israel, and the Ph.D. degree in electrical engineering and computer science in 2001 from the Massachusetts Institute of Technology (MIT), Cambridge.

From January 2002 to July 2002, she was a Postdoctoral Fellow with the Digital Signal Processing Group, MIT. She is currently an Associate Professor with the Department of Electrical Engineering, Technion-Israel Institute of Technology, Haifa. She is also a Research Affiliate with the Research Laboratory of Electronics, MIT. Her current research interests are in the general areas of signal processing, statistical signal processing, and computational biology. She was in the program for outstanding students at TAU from 1992 to 1996. In 1998, she held the Rosenblith Fellowship for study in Electrical Engineering, MIT, and in 2000, held an IBM Research Fellowship.

Prof. Eldar is currently a Horev Fellow of the Leaders in Science and Technology program at the Technion and an Alon Fellow. In 2004, she was awarded the Wolf Foundation Krill Prize for Excellence in Research, and the Andre and Bella Meyer Lectureship in 2005. She is a member of the IEEE Signal Processing Theory and Methods Technical Committee, and an Associate Editor for the IEEE TRANSACTIONS ON Signal PROCESSING.

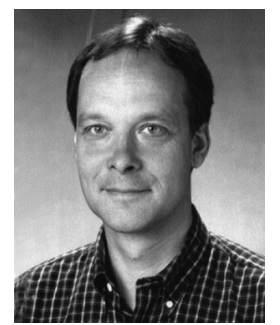

Michael Unser (M'89-SM'94-F'99) received the M.S. (summa cum laude) and Ph.D. degrees in electrical engineering from the Ecole Polytechnique Fédérale de Lausanne (EPFL), Lausanne, Switzerland, in 1981 and 1984, respectively.

From 1985 to 1997, he worked as a Scientist with the National Institutes of Health, Bethesda, MD. Currently, he is Professor and Director of the Biomedical Imaging Group at the EPFL. His main research area is biomedical image processing. He has a strong interest in sampling theories, multiresolution algorithms, wavelets, and the use of splines for image processing. He is the author of over 120 published journal papers in these areas.

Dr. Unser is the Editor-in-Chief of the Wavelet Digest, the electronic newsletter of the wavelet community. He has acted as Associate Editor or Member of the Editorial Board for nine other international journals, including the IEEE TRansactions on Medical IMAging, the IEEE Signal Processing Magazine, the IEEE TRANSACTIONS ON IMAGE PROCESSING (1992-1995), and the IEEE Signal Processing LeTters (1994-1998). He serves as regular chair for SPIE's Conference on Wavelets, which has been held annually since 1993. He was General Co-Chair for the first IEEE International Symposium on Biomedical Imaging (ISBI'2002), held in Washington, DC, July 7-10, 2002. He also chairs the newly created technical committee of the IEEE Signal Processing Society on Bio Imaging and Signal Processing (BISP). He received the 1995 and 2003 Best Paper Awards and the 2000 Magazine Award from the IEEE Signal Processing Society. 